DOE Tech. Memo. ARM VAP-002.1

\title{
The ARM Millimeter Wave Cloud Radars (MMCRs) and the Active Remote Sensing of Clouds (ARSCL) Value Added Product (VAP)
}

March 4, 2001

Eugene E. Clothiaux, The Pennsylvania State University

Mark A. Miller, Brookhaven National Laboratory

Robin C. Perez, Pacific Northwest National Laboratory

David D. Turner, Pacific Northwest National Laboratory

Kenneth P. Moran, NOAA Environmental Technology Laboratory

Brooks E. Martner, NOAA Environmental Technology Laboratory

Thomas P. Ackerman, The Pennsylvania State University

Gerald G. Mace, The University of Utah

Roger T. Marchand, The Pennsylvania State University

Kevin B. Widener, Pacific Northwest National Laboratory

Daniel J. Rodriguez, Lawrence Livermore National Laboratory

Taneil Uttal, NOAA Environmental Technology Laboratory

James H. Mather, The Pennsylvania State University

Connor J. Flynn, Pacific Northwest National Laboratory

Krista L. Gaustad, Pacific Northwest National Laboratory

Brian Ermold, Pacific Northwest National Laboratory

Work supported by the U.S. Department of Energy,

Office of Energy Research, Office of Health and Environmental Research 


\section{Abstract}

Over the past decade, the U.S. Department of Energy (DOE), through the Atmospheric Radiation Measurement (ARM) Program, has supported the development of several millimeter wavelength radars for the study of clouds. This effort has culminated in the development and construction of a 35-GHz radar system by the Environmental Technology Laboratory (ETL) of the National Oceanic and Atmospheric Administration (NOAA). Radar systems based on the NOAA ETL design are now operating at the DOE ARM sites located at the Southern Great Plains (SGP) Central Facility in central Oklahoma, on the islands of Nauru and Manus, Papua New Guinea, in the Tropical Western Pacific (TWP), and at Barrow on the North Slope of Alaska (NSA). These radars have come to be called the Millimeter Wave Cloud Radars (MMCRs). The importance of the MMCRs to the DOE ARM Program's strategy for remote sensing of clouds is outlined in Section 1.

The MMCRs are designed as a remote sensing tool that can accurately detect almost all of the hydrometeors present in the atmosphere. To illustrate the difficulty of this task, the various types of hydrometeors that can occur in the atmosphere are considered in the context of detection by the MMCRs (Section 1). Having outlined the nature of the remote sensing problem, a discussion ensues of the NOAA ETL design of the MMCR (Section 2). Next, we present the operational modes of the MMCRs (Section 3 ) and discuss them in some detail to illustrate the nature of the cloud products that are, and will be, derived from the MMCRs on a continuous basis.

The first set of products derived from MMCR data is based on detection of the significant returns in the data and subsequent classification of these detections as due either to clutter or to atmospheric hydrometeors. From these detections one can identify, as a function of time and height, regions of the atmosphere that contain hydrometeors. Using radar to conclusively identify regions of the atmosphere that do not contain any hydrometeors, such as $4 \mathrm{pm}$ radius cloud drops far from the radar, is generally not possible. With these limitations in mind, one can use significant radar detections, together with radar Doppler moments and/or spectra, lidar and passive radiation measurements, to estimate geometric boundaries of clouds, cloud water contents, and cloud particle sizes.

One effort for identifying significant returns in radar data and combining these detections with lidar estimates of cloud base height is called the Active Remote Sensing of CLouds (ARSCL) value added procedure (VAP). The outputs of this procedure are time-height maps of radar reflectivity, radar Doppler velocity, radar Doppler spectral width, and cloud base height estimates from laser ceilometers and Micropulse Lidars (MPLs). These products allow the geometric extent of clouds to be mapped and provide information on the distribution, size, and motions of the particles within cloud. The important elements of the ARSCL VAP are discussed in Section 4 and the products output by it are documented and illustrated in Sections 5 and 6. A reader who understands the contents of Sections 3 and 4 will be in a position to interpret the meaning, range of validity, and limitations of the ARSCL VAP and its products. The document ends with discussions of a few data quality issues (Section 7), of how to retrieve data from the archive (Section 8), and of some outstanding problems (Section 9). Appendices A1, A2, and A3 contain the ARSCL VAP input datastreams, flow chart, and product variable lists, respectively. 
To facilitate the discussion in this report, we make use of three articles on the MMCRs that are now in the peer-reviewed literature. Moran et al. (1998) give a general overview of the MMCRs, providing many illustrations of the cloud types that the MMCR was designed to study. The original four modes presented in Moran et al. (1998) are refined by Clothiaux et al. (1999), who both attempt to justify the slight changes to the original modes of Moran et al. (1998) and list the properties of the modes currently implemented at the ARM sites. Much of the discussion in Section 3 of this report attempts to present information necessary to understand all aspects of the description in Clothiaux et al. (1999). Finally, Clothiaux et al. (2000) contains a detailed description of the ARSCL VAP and its outputs.

For readers familiar with radar processing issues, Sections 5 and 6 and Appendices A1, A2, and A3 are perhaps of the greatest interest. In these parts of the document we describe the datastreams and variables produced by the ARSCL VAP (Section 5), those variables that are imaged (Section 6), ARSCL VAP input datastreams (Appendix Al), ARSCL VAP processing steps (Appendix A2), and the locations of the output variables in the datastreams (Appendix A3). Finally, Section 7 contains a list of known problems and should be inspected from time to time. Under the Derived Data Products link on the main ARM Web site (i.e., http://www.arm.gov), one can access the ARSCL VAP home page that contains quality updates and a bulletin board with an ongoing discussion of questions and problems. 


\section{Contents}

1. Introduction

2. MMCR System Components and Functional Characteristics ............................................ 3

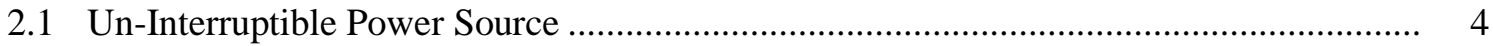

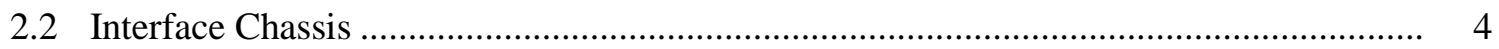

2.3 Intermediate Frequency Receiver/Modulator Chassis................................................. 4

2.4 Radio Frequency Coherent Up/Down Converter ..................................................... 4

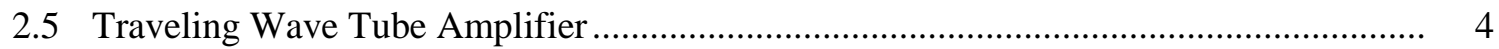

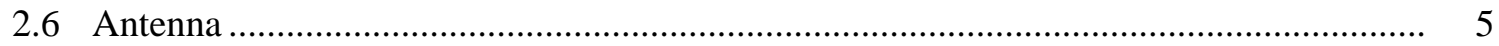

2.7 Transmit/Receive/Calibration Waveguide Sections ...................................................... 5

2.8 Pulse Controller.................................................................................................... 5

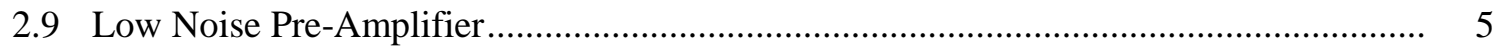

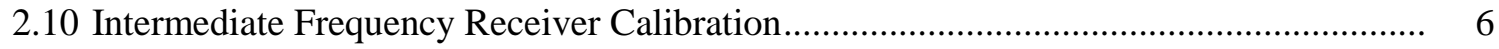

2.11 Multi-Channel System Monitor ............................................................................ 6

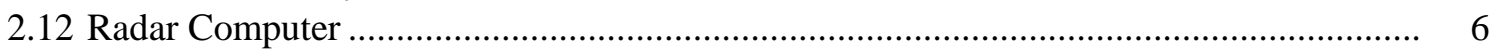

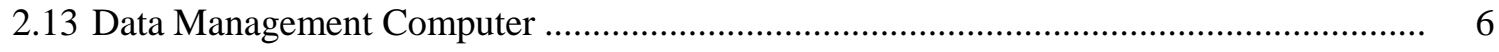

2.14 Data Tape System and Disk File Structure ................................................................... 6

3. MMCR Operational Modes: Tutorial ......................................................................... 7

3.1 Operational Parameters of the ARM MMCRs ................................................................ 7

3.2 Operational Modes of ARM MMCRs: Philosophy ...................................................... 14

3.3 Operational Modes of ARM MMCRs: Characteristics ............................................... 15

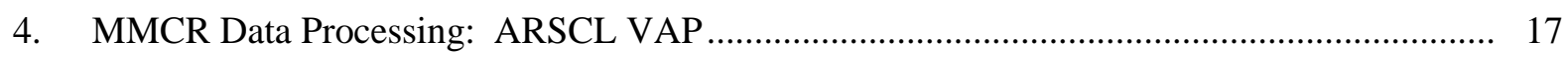

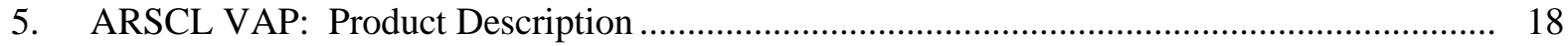

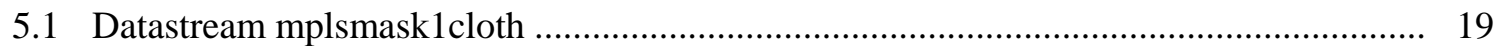

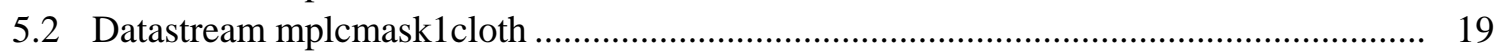

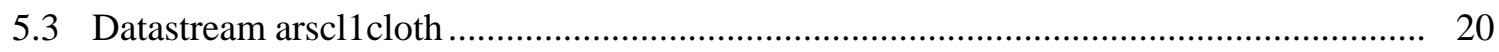

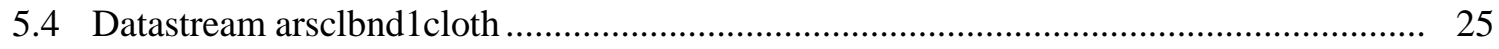

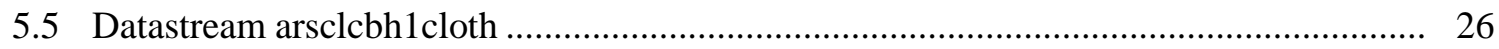

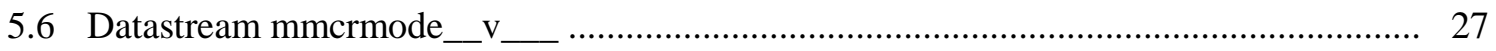

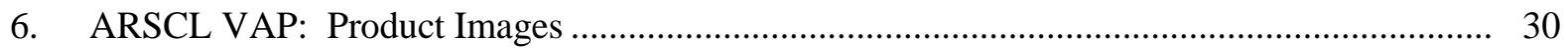

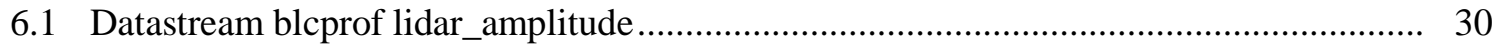

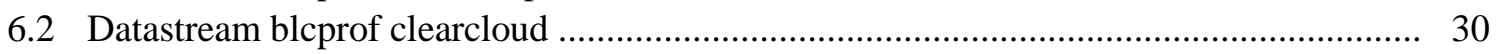

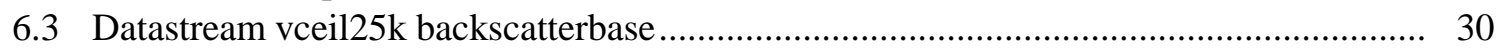

6.4 Datastream vceil25k backscatternobase .............................................................. 30

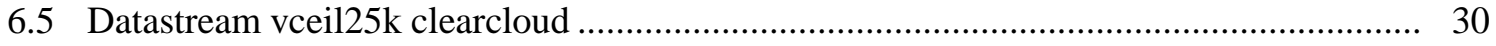

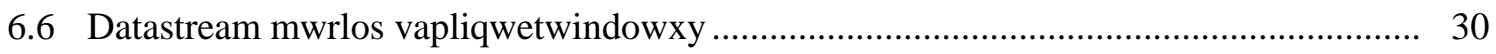

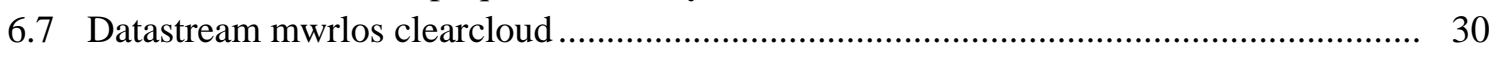




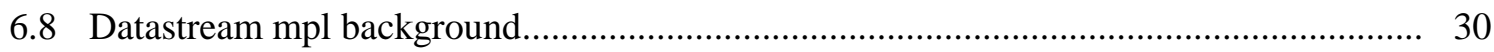

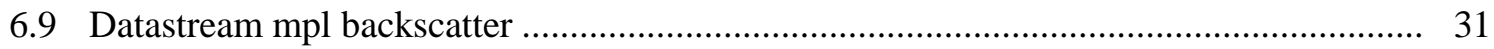

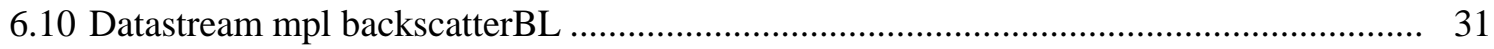

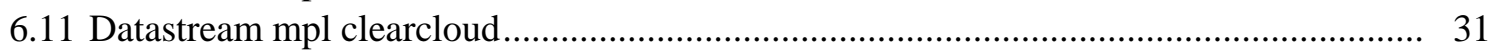

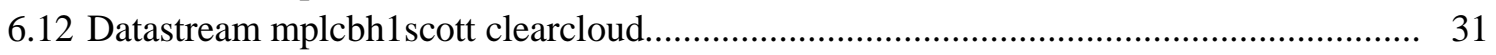

6.13 Datastream mplnor1camp backscatter................................................................. 31

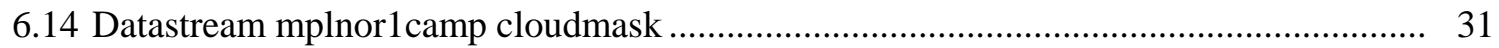

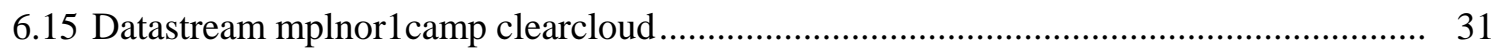

6.16 Datastream mplsmask1cloth SigniMaskMplCloth..................................................... 31

6.17 Datastream mplcmask1cloth C1oudMaskMplCloth .................................................... 31

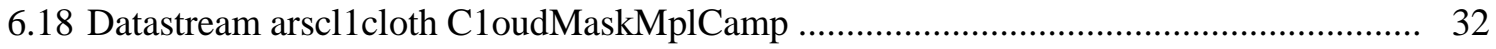

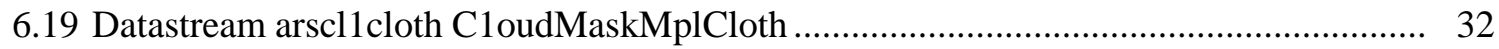

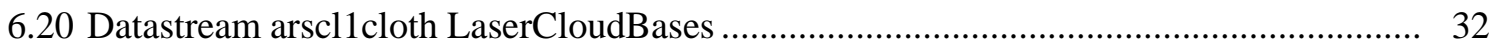

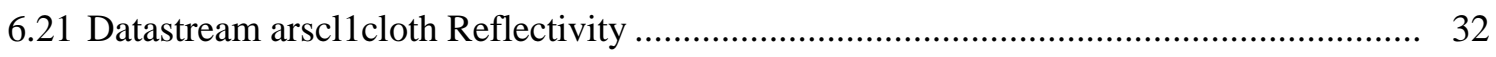

6.22 Datastream arscl1cloth ReflectivityNoClutter .......................................................... 32

6.23 Datastream arscl1cloth ReflectivityBestEstimate ....................................................... 32

6.24 Datastream arscl1cloth MeanDopplerVelocity ........................................................ 32

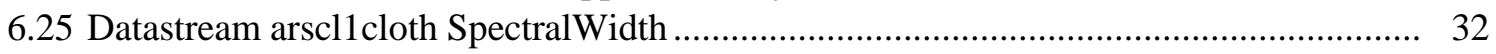

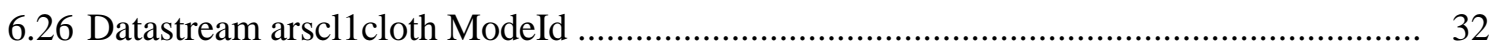

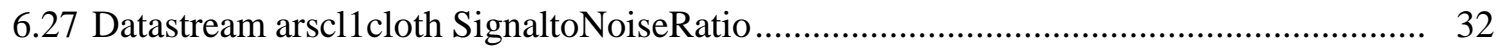

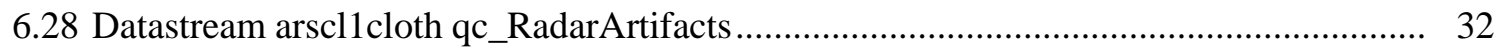

6.29 Datastream arscl1cloth qc_ReflectivityClutterFlag .................................................. 33

6.30 Datastream arsclbnd1cloth CloudLayerHeightsCloth ................................................ 33

6.31 Datastream arsclbnd1cloth qc_CloudLayerHeightsCloth ............................................ 33

6.32 Datastream arsclcbh1cloth LaserCloudBases ............................................................... 33

6.33 Datastream mmcrmode__ ___ 1 cloth Reflectivity .................................................... 33

6.34 Datastream mmcrmode__

6.35 Datastream mmcrmode_v__ 1cloth qc_RadarArtifacts ............................................. 33

6.36 Datastream mmcrmode__v__1cloth qc_ReflectivityClutterFlag.................................. 33

6.37 Datastream mmcrmode__v__ 1cloth qc_ReflectivityClutterFlagBL ............................. 33

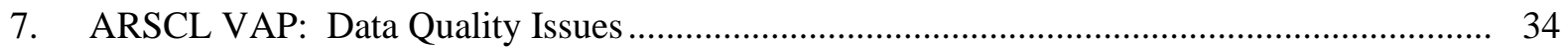

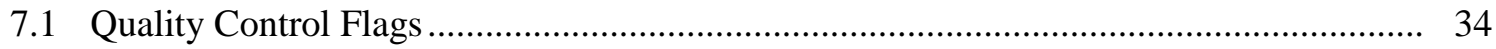

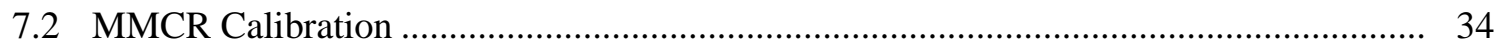

7.3 SGP Belfort Laser Ceilometer Height Offsets ........................................................ 35

7.4 NSA Micropulse Lidar Height Offsets................................................................... 35

8. ARSCL VAP: Retrieving Data from the ARM Archive ................................................. 35

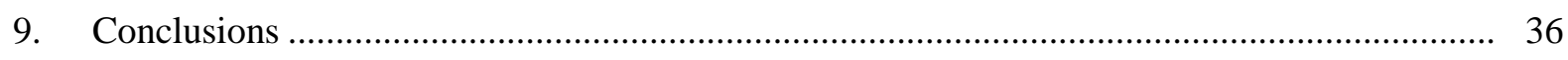

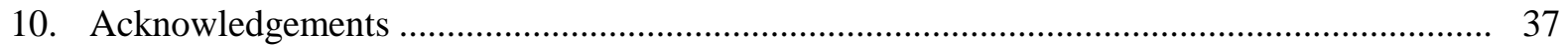

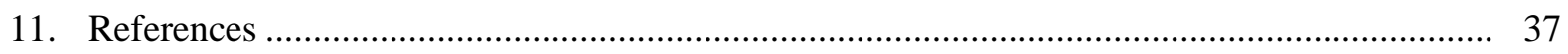




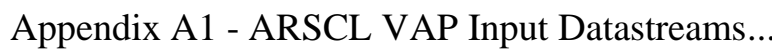

\section{Figures}

1 Block diagram of the NOAA ETL-designed ARM MMCR ................................................. 3

2 Dependencies between the operational parameters of the MMCR ......................................... 7

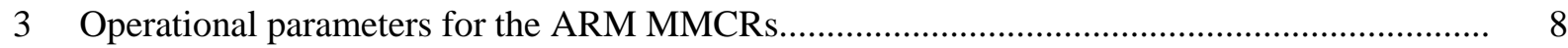

4 Schematic diagram illustrating the important steps in MMCR signal processing, from total particle backscatter cross section per unit volume to a power density spectrum generated from the MMCR "I" and "Q" voltage time series ....

5 Radar resolution volumes from which I and Q voltage sample pairs are generated depend upon the sampling strategy setup in the radar receiver

A1 Datastream inputs to the ARSCL VAP. 


\section{Introduction}

To fully understand the radiative impact of clouds on the climate system, both the macrophysical properties (i.e., horizontal and vertical distributions) and microphysical properties (i.e., particle shapes, sizes, and number concentrations) of clouds must be known. Whereas the horizontal distribution of clouds over the earth is presently characterized by passive radiometry from satellites, mapping the vertical distribution of clouds is currently best-achieved using lidars and radars. Lidars are able to detect all types of cloud particles in those circumstances when the lidar beam is able to penetrate to the location of the cloud particles. However, complete extinction of a lidar beam during heavy overcast, or a period with multiple cloud layers, is not uncommon. Millimeter wavelength radar observations are a natural complement to collocated lidar observations since radars operating at millimeter wavelengths can detect most cloud particles, and a millimeter wavelength radar beam generally penetrates all cloud types, except those that occur during periods of heavy rain. Radars operating at 35- and 94-GHz are the most utilized since the attenuation of the beam as a result of absorption by oxygen and water vapor is at a local minimum near these frequencies.

To provide the capability of mapping the vertical distribution of clouds in at least a few different climate regimes, the U.S. Department of Energy (DOE) Atmospheric Radiation Measurement (ARM) Program contracted the Environmental Technology Laboratory (ETL) of the National Oceanic and Atmospheric Administration (NOAA) to build a number of millimeter wavelength radars. The completely automated, continuously operating, vertically pointing, single-polarization, Doppler radars developed by NOAA ETL for the DOE ARM Program operate at a frequency of $34.86 \mathrm{GHz}$ (Moran et al. 1998). In the DOE ARM nomenclature, these radars are referred to as the Millimeter Wave Cloud Radars (MMCRs). Radar systems based on the NOAA ETL design are now operating at the DOE ARM sites located at the Southern Great Plains (SGP) central facility in central Oklahoma, on the islands of Nauru and Manus, Papua New Guinea, in the Tropical Western Pacific (TWP), and at Barrow on the North Slope of Alaska (NSA).

The vertical profiles of hydrometeor reflectivities and vertical velocities produced by the MMCRs are important to successfully realizing the ARM-stated goals of better understanding the impact of clouds on the radiation budget, improving the parameterizations of radiative transfer and clouds in numerical weather prediction and global climate models, and validating satellite retrievals. The Active Remote Sensing of Clouds (ARSCL) Value Added Product (VAP) comprise the current set of ARM algorithms for detecting the significant returns in the radar data and thereby ascertaining the distribution of the hydrometeors in the vertical column above the ARM sites. Once the cloud particles are detected, additional algorithms will be applied to these radar data in combination with additional active and passive sensor data to retrieve the microphysical properties of the hydrometeors above the sites. To successfully carry out these studies, an important scientific requirement is that the MMCRs must be able to detect the full spectrum of atmospheric hydrometeors.

To illustrate the range of reflectivities produced by atmospheric hydrometeors, consider examples of the radar reflectivity $(\mathrm{Z}$, in units of $\mathrm{dBZ}$ ) derived from radar observations and in situ measurements by aircraft in a number of different cloud conditions. For hydrometeors with dimensions smaller than the radar wavelength, $\mathrm{Z}\left[\mathrm{mm}^{6} \mathrm{~m}^{-3}\right]$ is defined by the integral 


$$
\int_{0}^{\infty} \mathrm{N}(\mathrm{D}) \mathrm{D}^{6} \mathrm{dD}
$$

where $\mathrm{D}[\mathrm{mm}]$ is the particle diameter and $\mathrm{N}(\mathrm{D})\left[\mathrm{m}^{-3} \mathrm{~mm}^{-1}\right]$ is the particle number density per size bin. The transform $10 \log _{10}(\mathrm{Z})$ casts $\mathrm{Z}$ into units of $\mathrm{dBZ}$.

Typical continental and marine boundary layer stratus values of $\mathrm{dBZ}$ are generally between approximately -40 and -25 dBZ (e.g., Figure 3 of Frisch et al. 1995; Figures 3 and 4 of Fox and Illingworth 1997). In stratocumulus (e.g., Noonkester 1984) and altocumulus (e.g., Heymsfield et al. 1991), the cloud drop mean diameters can be as low as 3-5 $\mu \mathrm{m}$ with liquid water contents as low as 0.01 $0.03 \mathrm{gm}^{-3}$. The radar reflectivities for these clouds can approach values as low as -55 to $-50 \mathrm{dBZ}$ (e.g., Figure 9 of Clothiaux et al. 1995). Values lower than $-55 \mathrm{dBZ}$ can occur if the clouds do not completely fill the volume of space sensed by the radar. (Note that the MMCR at the DOE ARM SGP site has a 0.2-degree beam width; consequently, the size of its sample volume at $5 \mathrm{~km}$ is approximately a cylinder with a diameter of $17.5 \mathrm{~m}$ and a height given by the radar sample volume length, typically $45 \mathrm{~m}$ or $90 \mathrm{~m}$.) Reflectivities from stratus cloud drizzle can range from values of $-15 \mathrm{dBZ}$ to more than $+20 \mathrm{dBZ}$ (e.g., Figure 3 of Frisch et al. 1995; Figure 3 of Fox and Illingworth 1997). In a comprehensive study of in situ aircraft measurements of cirrus ice particle sizes during FIRE I (the First ISCCP Regional Experiment), Atlas et al. (1995; Figure 12) concluded that the radar reflectivities of the cirrus ice particles would range from approximately -50 to $+10 \mathrm{dBZ}$.

Consequently, for the MMCRs to produce reliable estimates of the presence of all cloud types, they must be able to detect clouds with reflectivities that range from approximately -50 to $+20 \mathrm{dBZ}$, i.e., seven orders of magnitude in $\mathrm{Z}$, over the depth of the atmosphere. Designing a radar to perform accurately over a range of $10^{7}$ in power is a difficult task. The designers responded by providing flexibility in the operating modes of the radar (Section 2; Moran et al. 1998), such that when data from all of the operational modes are combined, an accurate depiction of the clouds in the vertical column above the radar emerges. Each mode is designed to detect particles with specific attributes and, as such, each mode involves tradeoffs between sensitivity and spatial/temporal resolution, sensitivity and range sidelobe artifacts, and velocity aliasing versus range aliasing. Note that the extreme sensitivity of the MMCR does not guarantee that all hydrometeors in an atmospheric column will be detected.

Four operational modes for the MMCRs are discussed in Section 3 that, when considered together, satisfy the requirement of detecting hydrometeors that range in reflectivity from -50 to $20 \mathrm{dBZ}$ (Clothiaux et al. 1999). To aid in understanding the logic behind the proposed four modes, Section 3 contains a description of each operational parameter of the MMCR and the impact of the parameter on the capability of the radar to detect certain types of hydrometeors. Each parameter is discussed in the context of a computer simulation of radar detections of cloud particles. Section 3 provides a fairly succinct description of radar processing that will enable one to understand the proposed operational modes and, consequently, the kinds of cloud data that will be derived from the MMCRs (Sections 4, 5, and 6). Before discussing the MMCR modes of operation, we present in Section 2 the system components that comprise the MMCR. 


\section{MMCR System Components and Functional Characteristics}

The basic operating characteristics of the NOAA ETL design of the MMCR are summarized in the article by Moran et al. (1998). A simple block diagram of the MMCR and its approximately 14 basic modules is illustrated in Figure 1. Following Figure 1, we provide a brief description of each module's characteristics and functionality. Fully comprehensive specifications for use in construction and maintenance, such as electronic schematic diagrams and information about component suppliers and manufacturers, are proprietary. Under provisions of NOAA's Cooperative Research and Development Agreement, future units of the MMCR are commercially available for sale or lease exclusively through Radian International, LLC.

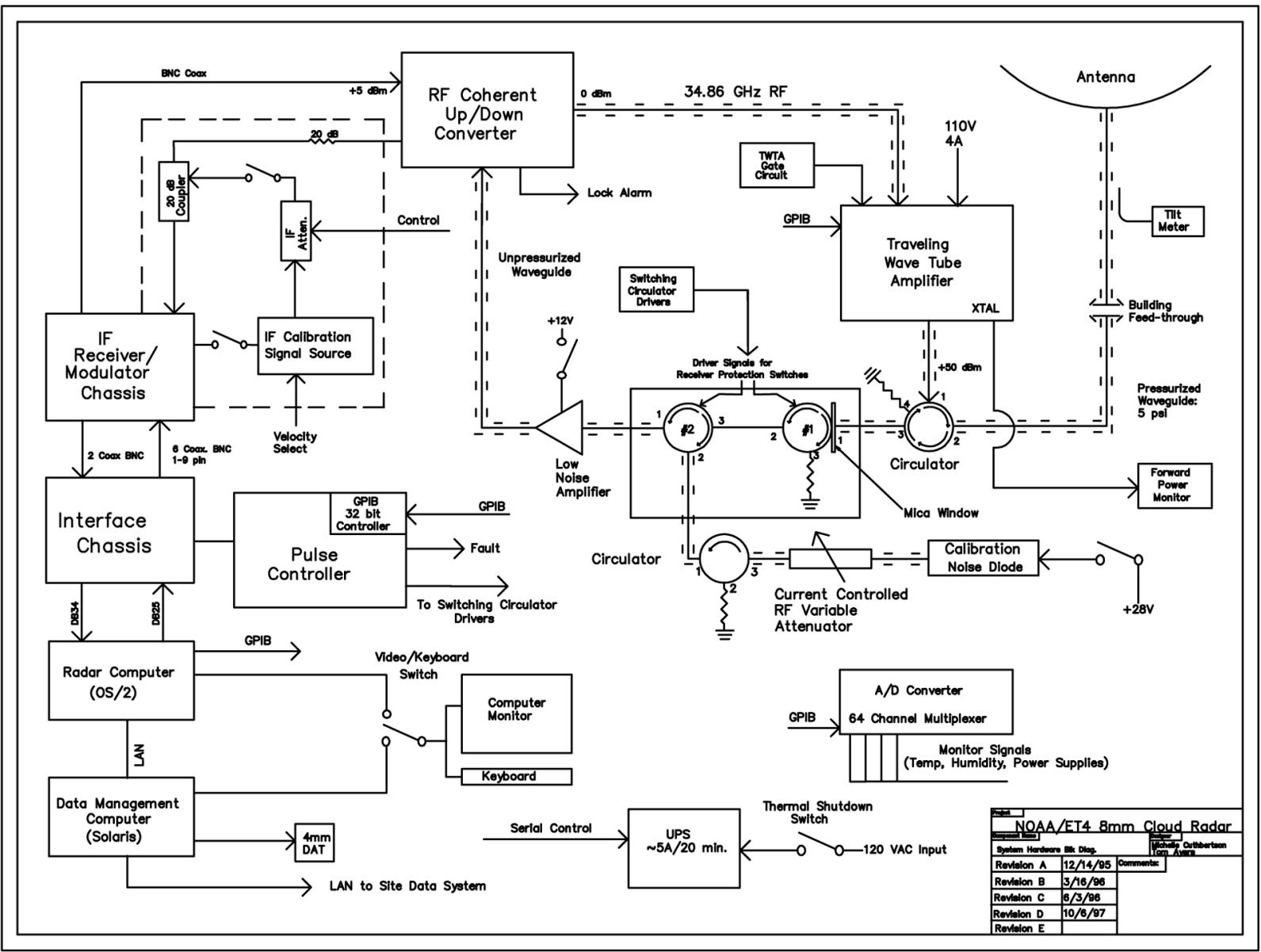

Figure 1. Block diagram of the NOAA ETL-designed ARM MMCR. 


\subsection{Un-Interruptible Power Source (UPS)}

A battery backup power source provides up to 12 amps of 12-volt AC power at $60 \mathrm{~Hz}$ for 20 minutes to all the radar electronics and computers, as well as to the traveling wave tube amplifier (TWTA) transmitter. Typical power usage is less than 9 amps, depending on the transmit duty cycle. The UPS provides an internal monitor that logs events and commands that are used for remote shutdown and reboot operations.

\section{$2.2 \quad$ Interface Chassis}

The two 10-bit analog-to-digital converters used in the in-phase (I) and quadrature (Q) channels of the receiver are housed in this chassis, along with the buffer amplifiers for the radar pulse control circuits. The analog input level for the converters is \pm 10 volts. Six binary computer-controlled digital output signals are provided for system control.

\subsection{Intermediate Frequency (IF) Receiver/Modulator Chassis}

The modulator consists of a pulse amplitude modulator and a binary phase modulator used for pulse coding. The intermediate frequency output signal is $60 \mathrm{MHz}$ at $+10 \mathrm{dBm}$. The receiver has a digitally controlled blanking circuit, a high gain IF amplifier and separate I and Q baseband channels with four computer selectable bandwidths. Each bandwidth has a separate analog gain control. The blanking circuits prevent the transient signals that are present near the transmit pulse from causing ringing in the receiver's Bessel filters. Isolation is better than $70 \mathrm{~dB}$. The IF and receiver amplifiers have a combined gain of about $72 \mathrm{~dB}$ with a noise figure of less than $12 \mathrm{~dB}$.

\subsection{Radio Frequency (RF) Coherent Up/Down Converter}

The $60-\mathrm{MHz}$ IF input signal is converted to the $34.86-\mathrm{GHz}$ RF output signal through a series of heterodyne mixers. The up converter uses a double heterodyne converter with local oscillator frequencies of 2.0 and $32.8 \mathrm{GHz}$. These are generated from a 100-MHz phase locked signal source with output frequencies of 2 and $16.4 \mathrm{GHz}$. The $32.8-\mathrm{GHz}$ signal is generated by a frequency doubler. The RF output level can be set by a front panel attenuator for TWTA saturation control up to $7 \mathrm{dBm}$. The $2-\mathrm{GHz}$ IF signal can de directed through an external delay line for receiver delay (range) calibration. The down converter uses the same IF frequencies to mix down to the $60-\mathrm{MHz}$ output. The downconversion process eliminates the unused side band noise. The total gain is about $7 \mathrm{~dB}$ and the noise figure is about $5 \mathrm{~dB}$. All RF signals use WR-28 waveguide.

\subsection{Traveling Wave Tube Amplifier (TWTA)}

The transmitter is a high-gain amplifier with excellent high-phase stability and low-amplitude ripple across the pulsed RF output. Peak RF power is $100 \mathrm{~W}$ with a maximum duty cycle of $25 \%$ for use with long pulse width, phase-coded waveforms. The tube is run in saturation and has several RF test ports used for monitoring the transmitted pulse for calibration. An IEEE-488 control bus operates the unit under computer commands: The RF output port is pressurized to 5 psi by an internal pump, which draws 
its dry air from a rear mounted desiccant bottle. The front panel has a digital display that monitors internal voltages, some of which are also available through the IEEE-488 bus. The unit has a protect circuit for single pulse shutdown in case of an output port RF mismatch.

\subsection{Antenna}

The antenna is a fixed position, vertically pointing parabolic dish with a rearmounted feed and Casegrain sub-reflector. A 10-foot diameter antenna, which has a gain of $57.2 \mathrm{~dB}$ and a beam width of $0.19^{\circ}$, is used on the Southern Great Plains (SGP) radar. The smaller 6-foot-diameter antennas have a gain of about $53.4 \mathrm{~dB}$ and a beam width of $0.29^{\circ}$ and are deployed at the North Slope of Alaska (NSA) and Tropical Western Pacific (TWP) Cloud and Radiation Testbed (CART) sites. First sidelobe levels are typically better than $18 \mathrm{~dB}$. The sub-reflector is mounted on four spars and an electrically conducting cylindrical shroud is attached to the edge of the dish. A custom fiberglass radome material, tuned for optimum transmission at the radar's frequency, is stretched across the shroud and held down with spring tensioners. On the antennas used in the tropics, a smaller blower and heater unit circulates the air inside the antenna chamber to reduce the chances of moisture condensation. A tilt-meter is attached to the antenna mount to measure any change in leveling.

\subsection{Transmit/Receive/Calibration Waveguide Sections}

The RF output of the TWTA is directed to the antenna through a low-loss waveguide circulator, which acts as a passive TR (transmit/receive) switch. The output waveguide section is pressurized to 5 psi. The received energy from the antenna is directed by the circulator to the receiver pre-amplifier through a series of active switching circulators. The switching circulators act as active isolators during the transmit pulse and have switching times of about $600 \mathrm{~ns}$. Each switch provides about $25 \mathrm{~dB}$ of isolation, protecting the sensitive pre-amplifier from damage. During a special calibration cycle, the switches can be used to inject a calibrated noise source into the receiver. An RF attenuator is automatically stepped through several levels to provide a measure of the calibration curve for the radar receiver/ processor.

\subsection{Pulse Controller}

The pulse controller contains the logic circuitry for the active pulse control of the switching circulators and the TWTA. It also contains the IEEE-488 logic control circuitry for the automatic calibration hardware, which includes the IF attenuator, the RF attenuator, and the noise diode.

\subsection{Low Noise Pre-Amplifier (LNA)}

The pre-amp is a high-gain, low-noise amplifier used to provide a high sensitivity receiver. The LNA has $33 \mathrm{~dB}$ of gain and a noise figure of about $3 \mathrm{~dB}$. The maximum input level of the LNA is about $+15 \mathrm{dBm}$. 


\subsection{Intermediate Frequency (IF) Receiver Calibration}

A separate IF signal source is provided that can be injected into the receiver to characterize the IF amplifiers as part of the calibration. The signal source is offset in frequency from the radars local oscillator to provide a received signal that has an equivalent Doppler velocity of $1 \mathrm{~m} \mathrm{~s}^{-1}$. The Doppler signal is attenuated by the IF attenuator, then processed to characterize the receiver.

\subsection{Multi-Channel System Monitor}

A 64-channel analog-to-digital converter (A/D) provides the monitor functions of the radar hardware. The A/D is connected to the IEEE-488 bus. It can monitor all the power supplies, internal temperature and humidity, external antenna tilt and temperature, several equipment temperatures, transmitted RF power, and several logical fault indicators. The hourly statistical results of the system monitoring are combined with the measured values from the UPS and reported in the hourly health message.

\subsection{Radar Computer}

Radar operations are controlled by a personal computer (PC) running under the OS/2 operating system. The radar control and processing software program, called POP, runs in a DOS window and several other tasks, including networking, system monitoring, and instrument control on the IEEE-488 bus, run as independent task windows. The radar task collects data every half hour and FTPs it to the data management computer. Extra disk space is allocated to store 2 weeks of data if network communications are lost.

\subsection{Data Management Computer}

Data collection, calibration, and display tasks run under the Solaris operating system on a PC dedicated to data management. Incoming radar data are converted to netCDF format and a special software program, called CONDOR, is available to play back a timeheight graphical image of the radar moment data. Calibration data from the RF noise diode are collected and converted into receiver calibration curves for the four operating modes. The calibration constants are applied to all incoming radar data to produce calibrated reflectivity measurements. An hourly health message is produced. Extra disk space is allocated to store 3 weeks of data in case the on-site data system cannot collect the available data.

\subsection{Data Tape System and Disk File Structure}

A 4-mm tape archives daily data sets as a secondary backup to the on-site data system archiver. Tapes normally hold about 7 weeks of data; monthly replacement is recommended. Original incoming radar files and all processed (calibrated) data sets are retained on disk in a separate managed file structure. The file manager maintains the most recent data sets and removes the oldest to keep the disk system at less than $70 \%$ capacity. Duplicates of incoming and processed data are available in an outgoing directory for capture by the on-site data system. 


\section{MMCR Operational Modes: Tutorial}

The following subsections present a detailed description of the MMCR's operational parameters.

\subsection{Operational Parameters of the ARM MMCRs}

The functionality of the ARM MMCRs is a consequence both of the hardware that is used to build them and of the control parameters that are used to run them. The hardware features of the radar, especially its operating wavelength of approximately $8.6 \mathrm{~mm}$, result from an attempt to maximize sensitivity to cloud particles while reducing the effect of atmospheric attenuation on the radar beam. For a given choice of radar hardware the sensitivity of the radar to cloud particles can be enhanced through the choice of operational parameters that are used to run it. The primary parameters in this regard are (1) the number of range sample volumes $\left(\mathrm{N}_{\mathrm{vol}}\right),(2)$ the range sample volume spacing $\left(\mathrm{R}_{\text {space }}\right)$, (3) the pulse width $\left(\tau_{\mathrm{pw}},\right)$, (4) the interpulse period $\left(\tau_{\mathrm{ipp}}\right),(5)$ the number of coded bits $\left(\mathrm{N}_{\mathrm{bits}}\right),(6)$ the number of coherent averages $\left(\mathrm{N}_{\text {coh }}\right)$, (7) the number of spectra averaged $\left(\mathrm{N}_{\text {spec }}\right)$, and (8) the number of fast Fourier transform (FFT) points $\left(\mathrm{N}_{\mathrm{fft}}\right)$. Once these operational parameters are set, other parameter values follow as a consequence (Figure 2). Examples of the current sets of operational parameters running on the ARM MMCRs are illustrated in Figure 3. Note that Figures 2 and 3 contain identical information to tables in Clothiaux et al. (1999). Furthermore, much of the following information in Section 3 is a tutorial on radar operation and the important information relating to the specifics of the ARM MMCRs can be found in a much more succinct form in Clothiaux et al. (1999). To elucidate the trade-offs between the important parameters in the operation of the ARM 35-GHz radars, or MMCRs, consider the schematic diagram in Figure 4. For the MMCRs, one goal is to detect all cloud hydrometeors in each volume of space (Figure 4a) that is probed by the propagating electromagnetic field within the radar pulse. For simplicity, the effects of the wind are neglected, and the velocities of the particles relative to the radar (i.e., the vertical velocities of the particles) as a result of gravity (Rogers et al. 1993) are computed. Cloud

\begin{tabular}{l}
\hline \multicolumn{1}{c}{ Radar Parameter Relationships } \\
\hline$R_{\text {max }}=R_{\text {deadtime }}+\left(N_{\text {vol }}-1\right) R_{\text {space }}$ \\
$R_{\mathrm{u}}=\left(c \tau_{\mathrm{ipp}}\right) / 2$ \\
$\Delta R=\left(c \tau_{\mathrm{pw}}\right) / 2$ \\
$V_{\mathrm{u}}=\lambda /\left(4 \tau_{\mathrm{ipp}} N_{\mathrm{coh}}\right)$ \\
$\Delta V_{\mathrm{u}}=\left(2 V_{\mathrm{u}}\right) / N_{\mathrm{fft}}$ \\
$T_{\mathrm{s}} \propto N_{\mathrm{vol}}, N_{\mathrm{fft}}, N_{\mathrm{spec}}, 1 /$ Processor Speed \\
Radar Sensitivity $\propto \tau_{\mathrm{pw}}, N_{\mathrm{bits}}$ \\
Receiver Noise $\propto 1 / N_{\mathrm{coh}}, 1 / \sqrt{N_{\mathrm{spec}}}$ \\
\hline
\end{tabular}

Figure 2. Dependencies between the operational parameters of the MMCR. 


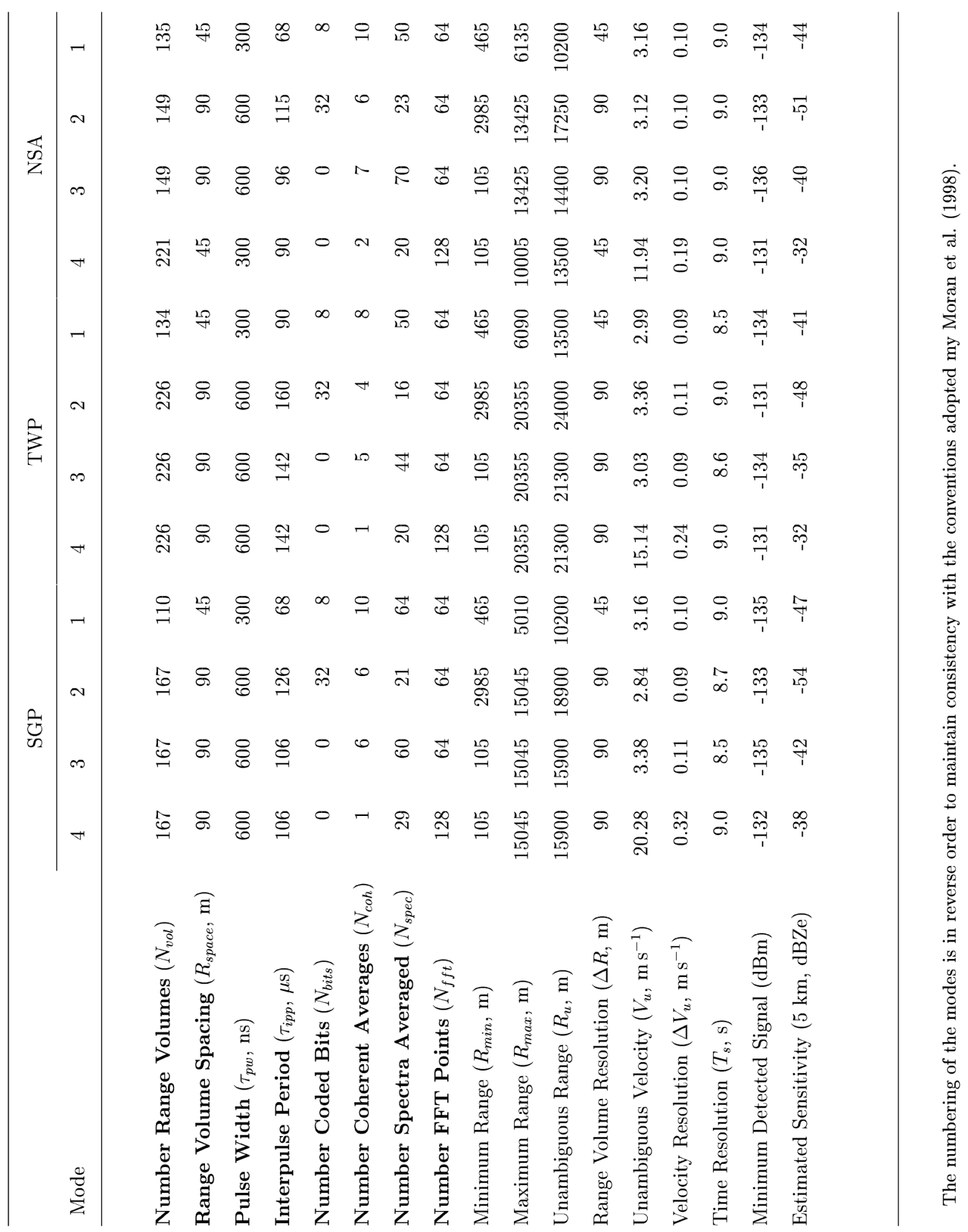

Figure 3. Operational parameters for the ARM MMCRs. 

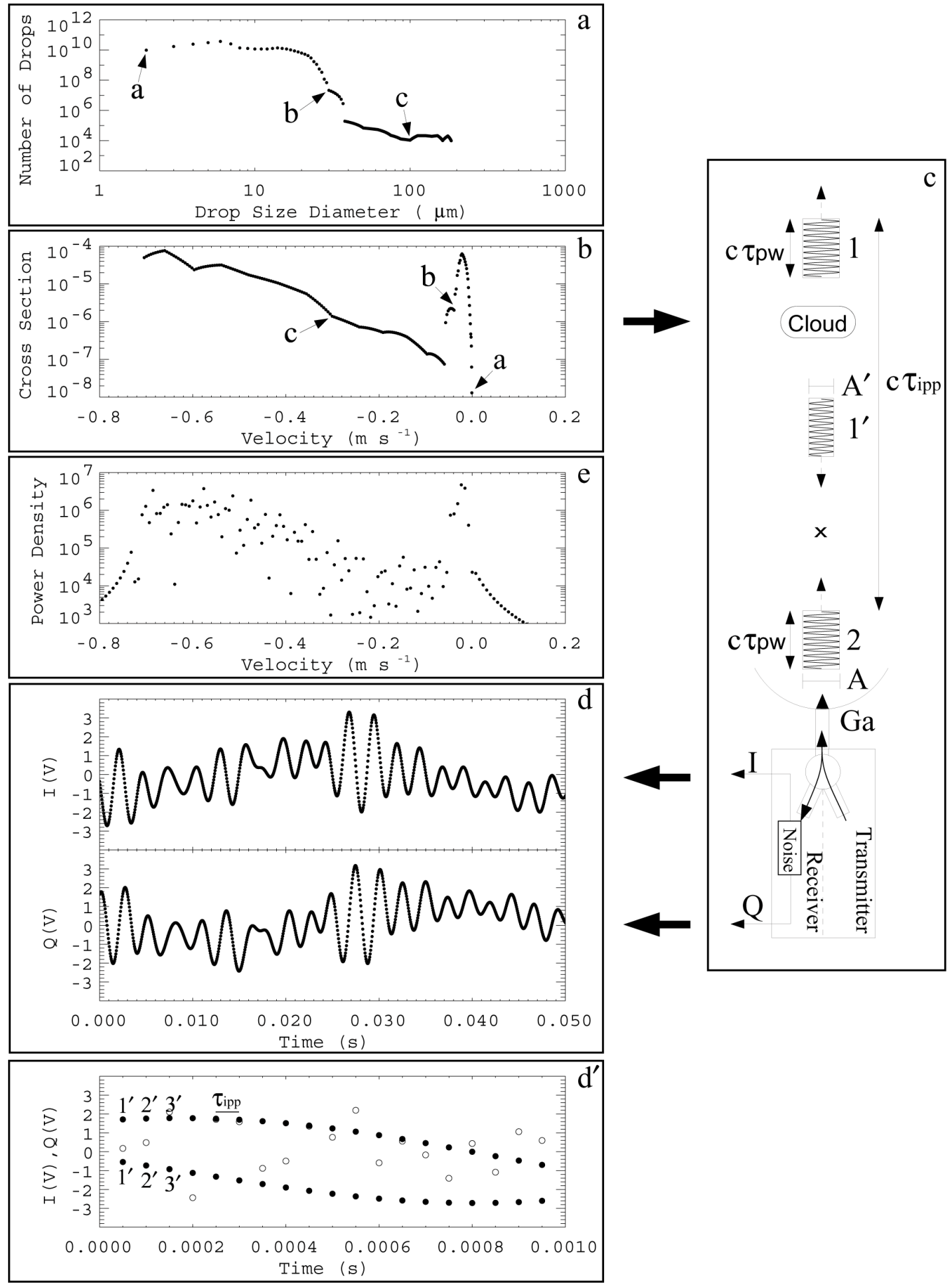

Figure 4. Schematic diagram illustrating the important steps in MMCR signal processing, from total particle backscatter cross section per unit volume to a power density spectrum generated from the MMCR "I" and "Q" voltage time series. (a) An example of the total number of drops per 
unit particle diameter in the radar sample volume as a function of drop diameter for a stratus cloud deck at approximately $2.2 \mathrm{~km}$. The radar resolution volume in this case was a $4014 \mathrm{~m}^{3}$ with a diameter of $7 \mathrm{~m}$ and a height of $90 \mathrm{~m}$. The total number of drops in the volume was 3.08 $\times 10^{11}$. (b) The total backscatter cross section of the cloud drops versus the vertical velocities of the drops. Cross sections were computed using Mie theory at a wavelength of $3.19 \mathrm{~mm}$, or 94$\mathrm{GHz}$. (c) Schematic diagram of the radar probing the cloud with pulses of electromagnetic energy. (d) The I and $Q$ voltages produced by the radar receiver as a result of the cloud; each radar pulse contributes to a pair of I and $Q$ voltages for each range gate. (d') A blow-up of the first $19(\mathrm{I}, \mathrm{Q})$ voltage pairs in d; the I voltages are represented by the upper group of solid circles; the $Q$ voltages are represented by the lower group of solid circles. Receiver noise is illustrated by the open circles. (e) The power density spectrum produced by applying a complex fast Fourier transform to the first 4096 I and $Q$ voltage pairs in d; the power density units are arbitrary and depend upon the radar calibration.

particles in the size range from $2 \mu \mathrm{m}$ to $29 \mu \mathrm{m}$ diameters fall with velocities from approximately $2.0 \mathrm{x}$ $10^{-4} \mathrm{~m} \mathrm{~s}^{-1}$ to $3.4 \times 10^{-2} \mathrm{~m} \mathrm{~s}^{-1}$, while particles with diameters over $162 \mu \mathrm{m}$ fall with velocities greater than $5.6 \times 10^{-1} \mathrm{~m} \mathrm{~s}^{-1}$. In each volume of space sensed by the MMCRs, the total backscatter cross section per radar resolution volume as a function of vertical particle velocity, called the Doppler spectrum, is the fundamental quantity obtained from the radar (Figure 4b). Although the total number of cloud particles greater than $100 \mu \mathrm{m}$ diameter is small compared to the total number of smaller particles, the larger particles nonetheless contribute most to the energy that is scattered back towards the radar; consequently, these larger particles often provide the dominant contributions to integrated quantities such as the reflectivity $(\mathrm{Z})$.

The MMCRs can be used to infer the distribution of particle backscatter cross section versus vertical Doppler velocity, as illustrated in Figure 4c. (See Battan [1973], Doviak and Zrnic [1984, 1993], Sauvageot [1992], Skolnik [1990], and Ulaby et al. [1982] for detailed discussions of these topics.) The transmitter delivers pulses of electromagnetic energy to the antenna, which directs the beam upwards into the vertical column of the atmosphere above the radar. In the schematic (Figure 4c), the power produced by the transmitter is represented by the amplitude (A) of the outgoing pulses and the spatial extent of the pulses is $\mathrm{c} \tau_{\mathrm{pw}}$, where $\mathrm{c}\left[\mathrm{m} \mathrm{s}^{-1}\right]$ is the speed of light and $\tau_{\mathrm{pw}},[\mathrm{s}]$ is the duration of the pulse, or the pulse width in time units. The phases of the outgoing electromagnetic waves are identical from pulse to pulse. The time interval between pulses, i.e., the interpulse period, is represented by $\tau_{\text {ipp. }}$ In Figure $4 \mathrm{c}$, the pulse labeled " 1 " has interacted with the cloud represented by the information in Figures $3 \mathrm{a}$ and $1 \mathrm{~b}$, leading to part of its energy, labeled by pulse " 1 "" with amplitude $A^{\prime}$, being scattered back towards the radar. The radar antenna with the gain $\mathrm{G}_{\mathrm{a}}$ collects some of this backscattered energy and routes it through the radar electronics to produce the received voltage time series.

For the MMCRs, the original received voltage time series is split into two identical time series. One of the two time series is subsequently delayed by a quarter wavelength and is processed to form the "Q" time series, whereas the remaining time series, identical in all respects to the original time series but with half the power, is processed to form the "I" voltage time series. The voltage pair I and Q (Figure 4d) represent a complex vector whose direction of rotation indicates the direction of motion of the target(s) with respect to the radar. 
For each pulse, a single pair of I and Q voltages is acquired for each distinct radar sample volume above the radar. The distance between radar sample volumes is called the range volume spacing $\left(\mathrm{R}_{\text {space }}\right)$. The spatial extent along the beam, or range resolution $(\Delta R)$, that potentially contributes to each pair of $I$ and $Q$ voltages equals $\left(c \tau_{\mathrm{pw}}\right) / 2$ (Figure 5). For the ARM radars, $\mathrm{R}_{\text {space }}$ is set equal to $\Delta \mathrm{R}$ (Figure $5 \mathrm{c}$ ). There is a minimum usable range $R_{\text {deadtime }}=c \tau_{\text {deadtime }}$ associated with the radar receiver deadtime $\left(\tau_{\text {deadtime }}\right)$, a short period of time during and following the pulse transmission process when the radar receiver is turned off in order to insure that no significant amount of the transmitter pulse power is directly reflected into the radar receiver. The maximum range $\left(\mathrm{R}_{\max }\right)$ is determined by the number of range resolution volumes $\left(\mathrm{N}_{\mathrm{vol}}\right)$ from which the radar receiver collects samples of the return power. If clouds are present above the unambiguous range $\left(\mathrm{R}_{\mathrm{u}}\right)$ of $\mathrm{c} \tau_{\mathrm{ipp}} / 2$ (typically, $\mathrm{R}_{\max }<\mathrm{R}_{\mathrm{u}}$ ), then the power returns from these clouds can potentially contaminate the power returns from clouds below $R_{u}$, as illustrated later in this section.

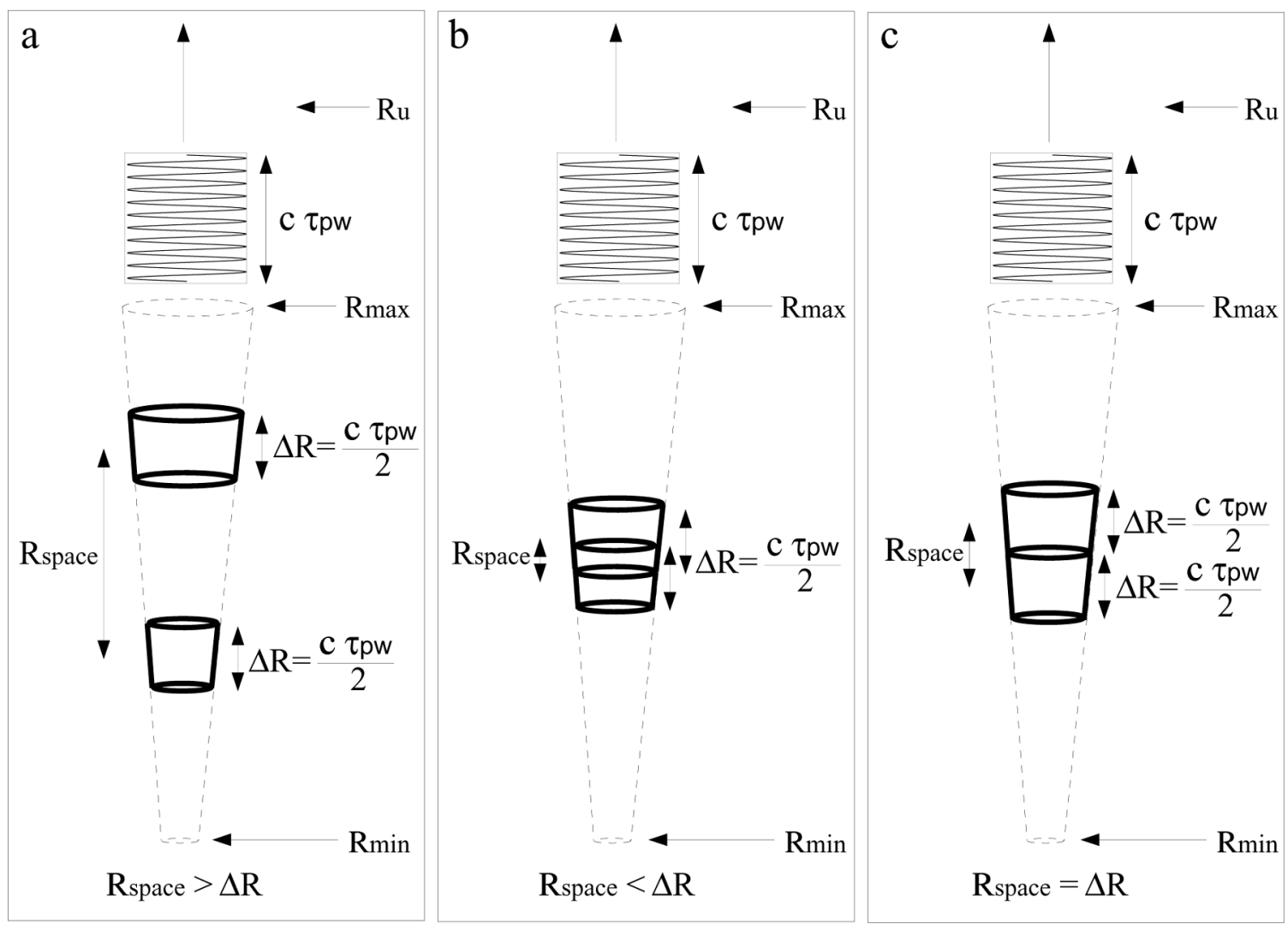

Figure 5. Radar resolution volumes from which I and $Q$ voltage sample pairs are generated depend upon the sampling strategy setup in the radar receiver. The vertical extent $\Delta R$ of the radar resolution volumes always equals $\mathrm{c} \tau_{\mathrm{pw}} / 2$, whereas the spacing $\mathrm{R}_{\text {space }}$ between radar resolution volumes is arbitrary. If $R_{\text {space }}>\Delta R$, there are regions in the atmosphere for which there are no corresponding I and $Q$ voltages. If $R_{\text {space }}<\Delta R$, I and $Q$ voltages corresponding to neighboring radar resolution volumes are not independent. For the ARM MMCRs, $R_{\text {space }}=\Delta R$. 
The variations of the I and Q voltages that correspond to a single radar sample volume in the region of the atmosphere that contains the cloud illustrated in Figure $4 \mathrm{c}$ are illustrated in Figure $4 \mathrm{~d}$. The I and Q voltages change in time (Figure $4 \mathrm{~d}^{\prime}$ ) as a result of the movement of the cloud particles relative to each other. The frequency content of the temporal variations in I and Q provide information about the cloud drop motions. To obtain information about the cloud drop motions, the I ("real part") and Q ("imaginary" part) voltage time series illustrated in Figure $4 \mathrm{~d}$ are input to a complex fast Fourier transform; the real output of the fast Fourier transform, called the power density spectrum, is illustrated in Figure 4e. The velocity axis in Figure $4 \mathrm{e}$ is obtained from the frequency output of the fast Fourier transform using the equation $v=\lambda f / 2$, where $v$ is the particle velocity, $\lambda$ is the wavelength of the radar and $f$ is the Doppler frequency shift as a result of particle motion.

The power density spectrum provides the information about the cloud drop motions that were originally presented in Figure $4 \mathrm{~b}$. If the radar produced only a single set of voltages, (e.g., the I voltages) a fast Fourier transform applied to the voltage time series would not result in any information on the direction of the particle velocities with respect to the radar. That is, when using fast Fourier transform methods, both the I and Q voltage time series are necessary to unambiguously determine which way, (i.e., up or down) the particles are moving with respect to the radar. Note that the variability of the power densities with velocity in Figure 4e is due to using a single realization of the drop spectrum to produce the figure. A smoother, less variable spectrum can be produced by averaging together (1) neighboring points in Figure $4 \mathrm{e}$ or $(2)$ consecutive spectra produced from the I and Q time series.

In the process of generating the I and Q voltage pairs, the receiver electronics also add noise to these voltages. The noise added to each voltage pair can be modeled as a random sample from a Gaussian distribution with a mean voltage of zero and a standard deviation dependent on the properties of the receiver electronics (Figure 4d'). For the example illustrated in Figure $4 \mathrm{c}$, the I and Q voltages corresponding to heights in the atmosphere that do not contain hydrometeors would contain noise voltages like those illustrated by open circles in Figure $4 d^{\prime}$. Otherwise, the receiver noise voltages are added to the I and Q voltages that result from the power returns from hydrometeors. The single most important hindrance to creating a usable spectrum, like that illustrated in Figure $4 \mathrm{e}$ (after suitable averaging), is the addition of receiver noise to the I and Q voltages. In fact, as the cloud reflectivities go below a certain level, the receiver noise contribution to the I and Q voltages makes detection of the atmospheric contribution to these voltages impossible.

There are four primary aspects of the radar hardware that affect radar sensitivity: (1) the radar wavelength, (2) the transmitter power, i.e., the magnitude of the transmitted electromagnetic field amplitude (A), (3) the gain $\left(G_{a}\right)$ of the antenna, and (4) the noise power generated by the radar receiver electronics. The radar wavelength is chosen in this case as a trade-off between maximizing the backscatter cross section of cloud particles, minimizing the effects of absorption by water vapor and oxygen, and obtaining a reasonable amount of transmitter power and antenna aperture at a relatively low cost. By increasing the transmitter power $(A)$ and the antenna gain $\left(\mathrm{G}_{\mathrm{a}}\right)$, the amplitudes of I and $\mathrm{Q}$ increase. If the amplitude of the noise generated in the receiver is reduced, the atmospheric contributions to I and Q are less contaminated and more easily identified. For the ARM radars, the radar electronics already consist of low-noise components, and the antennas are as large as is practical. Therefore, any future hardware-dependent enhancement in radar sensitivity will probably be a result of an upgrade to the 
radar transmitter. For a given set of radar hardware, radar sensitivity can, nonetheless, be enhanced by judicious choices of the operational parameters that are used to control the radar. Some options involved in setting up the radar parameters are now discussed, along with any drawbacks associated with them.

The I and Q voltage amplitudes that result from a cloud are proportional to the square root of the pulse width $\left(c \tau_{\mathrm{pw}}\right)$ when the cloud completely fills the sample volume of the radar. As cloud fills less of the radar sample volume, the magnitude of the power scattered back to the radar drops proportionately and the values of I and Q decrease. For geometrically thick cloud, the drawback to increasing the pulse width in order to enhance the return power is the increase of $\Delta \mathrm{R}$ (recall $\Delta \mathrm{R}=\mathrm{c} \tau_{\mathrm{pw}} / 2$ ) with a loss of spatial resolution within the cloud. To gain the added sensitivity of using a longer pulse width without a subsequent loss of spatial resolution, Moran et al. (1998) adopted the pulse coding techniques developed by Schmidt et al. (1979). To illustrate this technique, suppose the radar transmits a pulse width of $5760 \mathrm{~m}$, but with 32 changes in phase of the electromagnetic field within the radar pulse, breaking the pulse into 32 segments. A spatial resolution $\Delta \mathrm{R}$ of a single segment, in this case $(5760 \mathrm{~m} / 32) / 2$ or $90 \mathrm{~m}$, can be recovered by properly decoding the segment phases of the electromagnetic energy scattered back to the radar, with a consequent enhancement in sensitivity. The number of phase change encodings, in this case 32 , is called the number of coded bits and represented by $\mathrm{N}_{\text {bits. }}$. There are two important limitations to the pulse coding technique: (1) the first $\mathrm{N}_{\text {bits }}-1$ radar sample volumes are of limited value because they may contain inaccurate reflectivity estimates due to partial decoding and (2) regions of the atmosphere that have strong reflectivity gradients and large Doppler velocities may contaminate regions with weaker returns and smaller velocities, within a physical extent of $\mathrm{N}_{\text {bits }}-1$ radar sample volumes in either direction, as a result of imperfect segment decoding, called range sidelobe leakage.

Two methods for reducing the impact of noise utilize averaging of I and Q signal voltages and averaging of power density spectra. If hydrometeors in a radar sample volume do not move appreciably in the time $\mathrm{T}_{\mathrm{ipp}}$ that it takes two consecutive pulses from the radar to probe the radar resolution volume, then the atmospheric contributions to the I and Q voltages do not change much. (In Figure $4 d^{\prime}$, neighboring voltage samples, e.g., the voltages due to received pulses $1^{\prime}$ and $2^{\prime}$, are almost identical; in this case, $\tau_{\text {ipp }}=5 \times 10^{-5} \mathrm{~s}$ is sufficiently small to insure little relative movement of the drops between two consecutive radar pulses.) When the I and Q signal voltages do not change significantly from one pulse to the next, the I and Q voltages from consecutive pulses can be averaged together before being input to a complex fast Fourier transform; this averaging process is called coherent integration, or time-domain averaging. The standard deviation of noise in the power density spectrum is inversely proportional to the number $\left(\mathrm{N}_{\mathrm{coh}}\right)$ of consecutive I and $\mathrm{Q}$ samples that are averaged.

There are two limitations to coherent integration. First, the maximum unambiguous velocity of the power density spectrum is $\mathrm{V}_{\mathrm{u}}=\lambda /\left(4 \tau_{\text {ipp }} \mathrm{N}_{\text {coh }}\right)$, where $\lambda$ is the radar wavelength; hence, $\mathrm{V}_{\mathrm{u}}$ decreases as $\mathrm{N}_{\text {coh }}$ increases. If a particle has a vertical Doppler velocity that exceeds $V_{u}$, for example $V_{u},+0.2 V_{u}$ towards the radar, then there is velocity aliasing, or velocity folding, in the power density spectrum and the particle contribution to the power density spectrum appears at the incorrect velocity of $\mathrm{V}_{\mathrm{u}}-0.2 \mathrm{~V}_{\mathrm{u}}$ away from the radar. When velocity aliasing occurs in a power density spectrum, information about the vertical Doppler velocities of cloud particles is compromised. A second limitation in using coherent integration on the I and Q voltage time series is that the computed power density spectrum is weighted by a curve that has a steep roll off (i.e., a $3.9 \mathrm{~dB}$ or $60 \%$ loss) at the spectral ends near $\mathrm{V}_{\mathrm{u}}$. The weighting can shift 
the estimated mean velocity toward smaller values, as well as lead to lower estimates of the reflectivity, if the hydrometeor velocities are near $\mathrm{V}_{\mathrm{u}}$ and have a standard deviation (i.e., Doppler width) that is broad, covering many velocity bins. A practical way to reduce the influence of velocity weighting is to avoid having velocities near the spectral ends. This can be accomplished by reducing the number of coherent integrations $\left(\mathrm{N}_{\mathrm{coh}}\right)$, thus increasing $\mathrm{V}_{\mathrm{u}}$. Velocity weighting can also be removed entirely by not using any coherent integration (i.e., by setting $\mathrm{N}_{\text {coh }}=1$ ). In either case, however, a penalty is paid for decreasing $\mathrm{N}_{\text {coh }}$ : more noise and reduced sensitivity.

In principle $\tau_{\text {ipp }}$ could be made sufficiently small so that coherent integration would work for a broad range of $\left(\mathrm{N}_{\text {coh }}\right)$ values. However, as $\tau_{\text {ipp }}$ decreases, the problems with range aliasing, or second trip echoes, increase. Consider Figure 4c. If there is a cloud located at the position of the " $x$ " in the figure, then backscattered power from pulse 2 as a result of this cloud will arrive at the radar receiver at the same time as the backscattered power contained in pulse $1^{\prime}$, which originated from a cloud further away from the radar. In this case, the I and Q voltages as a result of the two clouds are superimposed. To eliminate this problem, $\tau_{\text {ipp }}$ must be sufficiently large to ensure that the radar does not emit a pulse until all of the backscattered energy from previous pulses has reached the radar.

Another method for reducing the impact of noise is to average consecutive power density spectra (e.g., Figure 4e but in units of $Z$ ). This type of averaging process is called incoherent integration or frequency domain averaging. To understand the implications of incoherent integrations, consider that for each radar pulse, two voltages (i.e., I and Q) are created for each of the $\mathrm{N}_{\mathrm{vol}}$ radar resolution volumes in the vertical column above the radar. Once the number of points $\mathrm{N}_{\mathrm{fft}}$ in each spectrum is set (and hence, the velocity resolution of the spectrum since the velocity change $\Delta \mathrm{V}$ between neighboring points in the power density spectrum is $\left.\Delta \mathrm{V}=\left(2 \mathrm{~V}_{\mathrm{u}},\right) / \mathrm{N}_{\mathrm{fft}}\right)$, the radar must transmit $\mathrm{N}_{\text {coh }} \mathrm{x} \mathrm{N}_{\mathrm{fft}}$ pulses and save the resulting $2 \mathrm{~N}_{\mathrm{vol}} \mathrm{N}_{\mathrm{fft}}$ voltages. Typically, coherent integration is done in hardware; therefore, only $2 \mathrm{~N}_{\mathrm{vol}}$ $\mathrm{N}_{\text {fft }}$, as opposed to $2 \mathrm{~N}_{\mathrm{vol}} \mathrm{N}_{\text {coh }} \mathrm{N}_{\mathrm{fft}}$, voltages must be saved. The radar processor must then compute $\mathrm{N}_{\mathrm{vol}}$ FFTs of $\mathrm{N}_{\text {fft }}$ points each to produce a single spectrum corresponding to each radar resolution volume. The exact choice of the number of consecutive spectra $\left(\mathrm{N}_{\text {spec }}\right)$ to be averaged for each radar resolution volume depends primarily upon specification of the desired time resolution $\left(\mathrm{T}_{\mathrm{s}}\right)$ of the final average spectrum. Reduction in the standard deviation of noise in this case is inversely proportional to the square root of $\mathrm{N}_{\text {spec. }}$ A summary of this relationship, as well as the others already discussed, is presented in Figure 2.

\subsection{Operational Modes of ARM MMCRs: Philosophy}

The primary goal of the ARM MMCRs is to produce long-term records of the vertical distribution of clouds over the ARM sites. The data must also have sufficient quality to be useful both in studies of the impact of clouds on the atmospheric radiation field and in cloud modeling work. On one hand, therefore, the radar must be operated in a manner that allows it to detect most, if not all, of the hydrometeors above it. On the other hand, however, the data generated by the radar must have few, if any, artifacts in it. These two requirements cannot be met by running the radar with one set of operational parameters. 
The ARM MMCRs must be able to sense hydrometeors that range in reflectivity from -50 to $+20 \mathrm{dBZ}$. This requirement motivated the development of a sequence of four operating modes, as illustrated in Figure 3, where each mode in the sequence can be adjusted according to the climatology of clouds over a particular site. The characteristics of each mode are aimed at accurately determining the reflectivities of certain kinds of hydrometeors: (1) a "robust mode" (Mode 4) that produces accurate reflectivities at all heights all the time; (2) a "general mode" (Mode 3) that is fairly sensitive to all cloud particles at all altitudes with no data artifacts, except during heavier precipitation; (3) a "cirrus mode" (Mode 2) that is tuned to detecting weakly reflecting mid- and higher-level ice clouds; and (4) a "boundary layer stratus mode" (Mode 1) that is tuned to detecting weakly reflecting lower-level liquiddroplet clouds. When considered together, these four modes appear to provide the required sensitivity while covering the full $70 \mathrm{~dB}$ dynamic range of reflectivities with few, if any, artifacts in the data. The modes in Figure 3 for the SGP site are based on the original set of four modes developed by Moran et al. (1998) and refined by Clothiaux et al. (1999), and the sets of modes for the TWP and NSA sites are natural extensions of the SGP set.

\subsection{Operational Modes of ARM MMCRs: Characteristics}

To illustrate the sensitivities of the four modes in each set, consider the minimum detectable signals that result from the SGP modes (Figures 1 and 3; Clothiaux et al. 1999). To generate these values for the minimum detectable signals, radar reflectivities within each range resolution volume were first averaged over one hour during clear-sky conditions. The standard deviations of reflectivity with respect to the mean reflectivity were then computed. To obtain an estimate of the minimum detectable signal for range resolution volumes above $4 \mathrm{~km}$, the mean and standard deviation of the reflectivities for each resolution volume were added together. Because no clouds were present during the averaging interval, the resulting means and standard deviations of the reflectivities are the result of receiver noise, at least for reflectivities from resolution volumes above $4 \mathrm{~km}$. The variability in the receiver noise is what makes identifying significant, but small, cloud power returns difficult. Below $4 \mathrm{~km}$, contributions from insects, dust, etc., to the radar signals are evident, as the Mode 4 results in Figures 1 and 3 (Clothiaux et al. 1999) illustrate. Best fit curves of the radar equation $P_{\min }(r)=R_{c} \eta \min (r) r^{-2}$, where $P_{\min }(r)$ is the minimum detectable power in the radar receiver, $R_{c}$ is the radar constant, and $\eta_{\min }(r)$ is the minimum detectable reflectivity at range $r$, to the data from heights above $4 \mathrm{~km}$ were then computed in order to extend the minimum detectable signals to all heights for which each mode is used. As the figures illustrate, from the surface up to approximately $10 \mathrm{~km}$, there is a mode available that can detect particles with reflectivities approaching values as low as $-50 \mathrm{dBZ}$.

There are a number of important points about these modes. Consider again the ARM SGP set as an example. The range resolution $\Delta \mathrm{R}$ and range spacing $\mathrm{R}_{\text {space }}$ of three modes are $90 \mathrm{~m}$, while the resolution and spacing of the boundary layer stratus mode are $45 \mathrm{~m}$. The minimum usable range $\mathrm{R}_{\min }$ for $\mathrm{SGP}$ Modes 4 and 3 is $105 \mathrm{~m}$, while for SGP Modes 2 and $1, R_{\min }$ is $2985 \mathrm{~m}$ and $465 \mathrm{~m}$, respectively, because of range sidelobe artifacts. The minimum value of $105 \mathrm{~m}$ results from the radar receiver deadtime that is necessary to protect the receiver electronics. The higher values of $R_{\min }$ for SGP Modes 2 and 1 result from pulse coding. For the pulse-coded modes, the data exist for sample volumes down to $105 \mathrm{~m}$; 
however, the data in these regions is difficult to interpret and is flagged as such. The maximum ranges $\mathrm{R}_{\max }$ are $15.045 \mathrm{~km}$ for SGP Modes 4, 3, and 2 and $5.010 \mathrm{~km}$ for SGP Mode 1. The temporal resolution $\mathrm{T}_{\mathrm{s}}$ of the data is currently $9 \mathrm{~s}$.

The temporal resolution is limited by the data processor of the radar and not the radar hardware, so enhancements in temporal resolution are possible. A new version of the radar processor is planned that will significantly improve the performance of the radar. This processor will provide two major benefits. First, coherent integration of neighboring I/Q voltages can be phased out in favor of FFTs with more points and subsequent averaging of neighboring FFT points; this eliminates the spectral weighting that time series integration imposes and it increases $V_{u}$, thereby reducing the chances of folded velocity measurements. Second, increased processor speed will mean either that more radar data can be processed during the same time interval, thus improving the overall system sensitivity for the same temporal resolution, or that the same amount of data can be processed at a much faster rate, thus improving the temporal resolution without sacrificing sensitivity.

As previously mentioned, the characteristics of each mode are tuned to accurately determine the reflectivities of various kinds of hydrometeors. For example, SGP Mode 4 (i.e., "the robust mode," Figure 4a of Clothiaux et al. [1999]) has an unambiguous velocity $\left(\mathrm{V}_{\mathrm{u}}\right)$ of $\pm 20.28 \mathrm{~m} \mathrm{~s}^{-1}$, which should be sufficient to cover the vertical Doppler velocities of the hydrometeors in most conditions, except possibly for strong convective storms when attenuation from heavy rain is also likely to be a limiting factor. For this mode, there is no coherent averaging (i.e., $\mathrm{N}_{\text {coh }}=1$ ) and the number of coded bits $\left(\mathrm{N}_{\text {bits }}\right)$ is 0 . Therefore, SGP Mode 4 is the least sensitive mode; however, when hydrometeors are detected by this mode, both the reflectivities and vertical Doppler velocities of the hydrometeors inferred from the data are uncontaminated and unaliased. SGP Mode 3 (i.e., "the general mode," Figure 4b of Clothiaux et al. [1999]) is a slight modification of SGP Mode 4. For SGP Mode 3, the number of coherent averages $\left(\mathrm{N}_{\text {coh }}\right)$ is 6 and the number of incoherent averages, or number of spectra averaged $\mathrm{N}_{\text {spec }}$ is 60 . SGP Mode 3 is approximately $5 \mathrm{~dB}$ more sensitive than SGP Mode 4 and should be able to detect most nonprecipitating clouds from the ground up to the tropopause. There is a drawback to SGP Mode 3: it has a relatively low unambiguous velocity $\left(V_{u}\right)$ of $\pm 3.38 \mathrm{~m} \mathrm{~s}^{-1}$, and when particles have vertical Doppler velocities outside of this range, the reflectivities of SGP Mode 3 are no longer valid because of coherent averaging and velocity weighting. However, particles with vertical Doppler velocities greater than \pm 3.38 $\mathrm{m} \mathrm{s}^{-1}$ are relatively large, and SGP Mode 4 should accurately measure the reflectivities of these particles. Therefore, SGP Mode 4 can be used to identify problems with data from SGP Mode 3.

The purpose of the last two modes is to detect low reflectivity clouds both in the boundary layer and in the middle and upper levels of the troposphere. Since low reflectivity clouds tend to be composed of particles with vertical velocities no greater than $1 \mathrm{~m} \mathrm{~s}^{-1}$ to $2 \mathrm{~m} \mathrm{~s}^{-1}$, both of the remaining two modes have a relatively low unambiguous velocity $\left(\mathrm{V}_{\mathrm{u}}\right)$. SGP Mode 2 (Le, "the cirrus mode," Figure 4c of Clothiaux et al. [1999]), though similar to SGP Mode 3, is approximately $10 \mathrm{~dB}$ more sensitive than SGP Mode 3 because of pulse coding (i.e., $\mathrm{N}_{\text {bits }}=32$ ). Pulse coding, however, leads to unusable reflectivities below $2985 \mathrm{~m}(105 \mathrm{~m}+32$ x $90 \mathrm{~m})$. Perhaps most importantly, for clouds between $3 \mathrm{~km}$ and $15 \mathrm{~km}$, this mode approaches a sensitivity of between -55 and $-45 \mathrm{dBZ}$ and, hence, should detect all but the most tenuous altostratus and cirrus, or clouds composed of extremely small particles. 
For good sensitivity in the boundary layer, pulse coding is required. To restrict the partially decoded reflectivity data to altitudes close to the surface in SGP Mode 1 (i.e., "the boundary layer stratus mode," Figure $4 d$ of Clothiaux et al. [1999]), the spatial resolution $(\Delta R)$ and the sample volume spacing $\left(R_{\text {space }}\right)$ are set to $45 \mathrm{~m}$ and the number of coded bits $\left(\mathrm{N}_{\text {bits }}\right)$ is set to 8 . The enhanced spatial resolution in the lower atmosphere is primarily driven by scientific interest in the structure of boundary layer stratus. In SGP Mode 1, contaminated reflectivities are limited to the lowest $465 \mathrm{~m}$; from 465 to $5010 \mathrm{~m}$, the mode has a sensitivity between approximately -60 to $-45 \mathrm{dBZ}$. As for SGP Mode 3, data from SGP Modes 2 and 1 can be flagged as suffering from complications of coherent averaging and range aliasing using SGP Mode 4 results. Compositing good data from these four modes leads to a complete description of the clouds in the vertical column above the site.

\section{MMCR Data Processing: ARSCL VAP}

The ARM SGP, TWP-Manus, TWP-Nauru, and NSA sites each have a suite of operational active remote sensors that consist of a Belfort or Vaisala laser ceilometer, an MPL, and an MMCR. The ARSCL VAP combines the data from these active remote sensors to produce a time series of the vertical distribution of cloud hydrometeors over the ARM sites. Since Clothiaux et al. (2000) describe the ARSCL VAP in detail, we do not present a detailed discussion of it here. Rather, we give a brief overview of the important elements of the ARSCL VAP, including a description of the inputs to the VAP and, perhaps more importantly, its output products.

The inputs to the ARSCL VAP are listed in Figure A1 (Appendix A1) and a flow chart that includes the processes to which the inputs are applied is illustrated in the flow chart contained in Figure A2 (Appendix A2). Because the flow chart illustrates the important elements of the ARSCL VAP, the current discussion centers around it. The symbols in the flow chart have the following meaning: (1) open circles represent one of the input datastreams represented in Figure A2, (2) open squares represent an algorithmic process that implements some of the ARSCL VAP functionality, (3) dark gray circles represent intermediate files kept either in internal memory as arrays or in netCDF files on a hard disk, and (4) light gray circles represent the netCDF output files generated by the ARSCL VAP.

The starting point for the ARSCL VAP is the MMCR power returns (in units of $\mathrm{dBm}$ ) for each mode that are contained in the mmcrcal.a1 file for a day (Figure $\mathrm{A} 2 \mathrm{~A}_{\mathrm{i}}, \mathrm{i}=1,2,3,4$, where the i subscript identifies the mode). We apply a "Cloud Mask Code" algorithm to these power returns on a mode-by-mode basis that attempts to identify all of the significant powers in the radar returns; the intermediate outputs of the cloud masking operation are labeled Figure $\mathrm{A}_{2} \mathrm{~B}_{\mathrm{i}}$. The ARSCL VAP algorithm then combines the reflectivities (dBZ) contained in the mmcrcal file (Figure $A 2 \mathrm{~A}_{\mathrm{i}}$ ) with the Doppler velocity $\left(\mathrm{m} \mathrm{s}^{-1}\right)$ and spectral width $\left(\mathrm{m} \mathrm{s}^{-1}\right)$ data contained in the corresponding mmcrmoments file (Figure $\mathrm{A} 2 \mathrm{~A}_{\mathrm{i}}$ ) for only those times and heights for which there is a significant detection in the cloud mask intermediate file (Figure $A 2 B_{i}$ ). The outputs of this process are the intermediate files labeled Figure $A 2 C_{i}$ that exist in memory.

Because each of the four modes may contain unique information, to build a comprehensive description of the atmospheric hydrometeors over the ARM sites the data from the four modes must be combined, or merged, together. The current criteria implemented in the "Merge Modes Code" algorithm 
are described by Clothiaux et al. (2000) and the intermediate netCDF files that this code produces are labeled Figure $A 2 D_{i}$. As Clothiaux et al. (1999) indicate, some of the data in each mode might not be accurate and these data can contaminate the merged data product represented by Figure $\mathrm{A}_{2} \mathrm{D}_{1}$. Consequently, we use the merged cloud data (Figure $\mathrm{A}_{2} \mathrm{D}_{1}$ ) to go back and search the individual mode data for problems. The updated mode data with flags indicating problem data are contained in the intermediate products labeled Figure $\mathrm{A} 2 \mathrm{~F}_{\mathrm{i}}$. These corrected mode data are again merged together to produce the final merged dataset represented by Figure $A 2 G_{1}$. The files represented by Figure $A 2 G_{1}$ are in netCDF and they contain our best estimate of the significant reflectivities in the MMCR data.

Since many of the significant reflectivities in the MMCR data are not from hydrometeors, but rather low density, airborne particulate/biological particles of some sort, we use the ceilometer and lidar data, which appear not to detect these airborne particles, to filter the MMCR data. To this end, we use the ARM standard ceilometer (blcstd, vcei125kstd) and MPL (mplcamp, mplscott) products represented by Figure $\mathrm{A}_{2} \mathrm{H}_{\mathrm{i}}(\mathrm{i}=1,2,3)$. We also apply an in-house "Cloud Mask Code" algorithm (Clothiaux et al. 1998), which is not dependent on an absolute calibration of the laser systems, to the raw ceilometer and MPL data (Figure $\mathrm{A} 2 \mathrm{H}_{0}$ ) to produce additional estimates (blccloth, vceil25kcloth, mplcloth) of the location of the atmospheric hydrometeors (Figure $\mathrm{A} 2 \mathrm{H}_{\mathrm{i}}{ }^{\prime}$ ). (Note that the in-house algorithm has yet to be implemented on the blc or vceil25k datastreams; this will only be done if there is a pressing need to do so and the backscatter data become readily available.) Since the laser-based algorithms often fail during periods of heavier precipitation, we use the wet window flag from the mwrloslilj datastream or the precipitation rate from the smetstd datastream (Figure $\mathrm{A} 2 \mathrm{I}_{2}$ ) to identify periods of precipitation. When all the laser-based retrievals of cloud base height fail during a precipitation event, the cloud base height is assigned a value of $0 \mathrm{~m}$.

All the laser-derived cloud base height data is input to the "Clear vs. Cloud Code" algorithm and a single, best-estimate of the cloud base height is derived. Using the best estimate cloud base heights, we apply a "Clutter Profile Code" algorithm to the merged MMCR data (Figure A2 $\mathrm{G}_{1}$ ) in order to characterize the clutter in the MMCR significant detections. (See Clothiaux et al. [2000] for a detailed description of this process.) Using the profiles of MMCR significant reflectivities generated by the "Clutter Profile Code" that characterize the clutter (Figure A2 $\mathrm{J}_{1}$ ), we attempt to identify the clutter in MMCR data using the "Remove Clutter Code." Once the clutter mask is created from the $\mathrm{G}_{1}$ datastream, it is used to filter the mode data represented by Figure $A 2 F_{i}$. The final netCDF output products are represented by Figure $A 2 K_{i}(i=1,2,3,4,5)$. The product labeled $K_{1}$ contains the merged, best-estimate locations of the atmospheric hydrometeors, while the products labeled $K_{i}(i>0)$ contain the original MMCR data together with information (i.e., a set of flags that assists in interpretation of these data).

\section{ARSCL VAP: Product Description}

The scope of the ARSCL VAP is well summarized by its inputs (Appendix A1) and outputs (Sections 5 and 6, as well as Appendices A2 and A3). The inputs, as Appendix A1 illustrates, are from laser ceilometers, which yield cloud base height estimates, MPLs, which provide estimates of cloud base height and vertical profiles of hydrometeors to the level where the lidar beam is completely attenuated, and MMCR reflectivities, mean Doppler velocities, and Doppler spectral widths. During periods of heavier precipitation, the laser retrievals of cloud base height often fail. Consequently, we use an indicator of 
surface precipitation obtained either from the Microwave Radiometer (MWR) wet window sensor or from an optical rain gauge to help identify these periods. The ARSCL VAP netCDF output files and the variables they contain are now briefly described. Motivation for these output files and variables are provided in Sections 3 and 4, and the netCDF files headers are provided in Appendix A3.

\subsection{Datastream mplsmask1cloth}

Outputs of the Clothiaux et al. (1998) MPL cloud detection algorithm include cloud base height and two time-height arrays, one indicating each significant detection and the other labeling those subset of significant detections that are from cloud. Significant detections and the subset of them labeled as cloud are very much dependent on the lidar cloud mask algorithm, so one should pay careful attention to the algorithm that is used to produce them. Datastream mplsmasklcloth contains the significant detection time-height array.

\subsection{1 base_time}

ARM standard time of the first record in seconds from the beginning of 1970.

\subsection{2 time_offset}

ARM standard time of the number of seconds from the base_time.

\subsubsection{Heights}

The distance [in meters] from the surface (i.e., MPL location) to the center of each MPL range bin.

\subsubsection{SigniMaskMpICloth}

The significant detection time-height array as produced by the Clothiaux et al. (1998) MPL cloud detection algorithm.

\subsection{Datastream mplcmask1cloth}

Outputs of the Clothiaux et al. (1998) MPL cloud detection algorithm include cloud base height and two time-height arrays, one indicating each significant detection and the other labeling those subset of significant detections that are from cloud. Significant detections and the subset of them labeled as cloud are very much dependent on the lidar cloud mask algorithm, so one should pay careful attention to the algorithm that is used to produce them. Datastream mplcmasklcloth contains those significant detections that are labeled as cloud by the Clothiaux et al. (1998) algorithm.

\subsection{1 base_time}

ARM standard time of the first record in seconds from the beginning of 1970. 


\subsection{2 time_offset}

ARM standard time of the number of seconds from the base_time.

\subsubsection{Heights}

The distance [in meters] from the surface (i.e., MPL location) to the center of each MPL range bin.

\subsubsection{CloudMaskMpICloth}

The subset of significant detections SigniMaskMplCloth labeled as cloud by the Clothiaux et al. (1998) MPL cloud detection algorithm.

\subsection{5 qc_BeamAttenuationMpICloth}

An experimental variable composed of the base 10 logarithm of signal power above cloud to clear-sky signal power at the same height. The hope is that this variable can be used to assess the degree of lidar attenuation through a cloud.

\subsection{Datastream arscl1cloth}

The primary output datastream of the ARSCL VAP. Because the data associated with the four MMCR modes occur at different times, we interpolate these data to a $10 \mathrm{~s}$ grid in time and a $45 \mathrm{~m}$ grid in height. The data contained in the current datastream is on this time-height grid. We are not sure if the data contained in this datastream are useful in time series analysis. However, these data do represent our best-estimate of the vertical locations of the cloud hydrometeors above the ARM sites. The laser-derived cloud masks are also interpolated to this grid; however, we do not interpolate the laser-derived cloud base heights to this grid since such an interpolation is meaningless. Clothiaux et al. (2000) describe a more meaningful way of moving the laser-derived cloud base heights to this grid.

\subsection{1 base_time}

ARM standard time of the first record in seconds from the beginning of 1970.

\subsection{2 time_offset}

ARM standard time of the number of seconds from the base_time.

\subsubsection{Heights}

The distance (in meters) from the surface (i.e., MMCR location) to the center of each range bin in the merged cloud product $45 \mathrm{~m}$ grid. 


\subsubsection{Reflectivity}

Radar reflectivity (0th Moment) in dBZe from all heights, which the ARSCL VAP algorithm processing has indicated as containing a significant power return, including power returns from insects and other clutter sources.

Note: This quantity is equivalent to the total area under the curve in Figure $4 b$ and is derived from measured Doppler spectra similar to the simulated spectrum in Figure $4 e$.

\subsubsection{ReflectivityNoClutter}

Radar reflectivity (0th Moment) in dBZe from all heights, which the ARSCL VAP algorithm processing has indicated as containing hydrometeors ONLY. Regions that contain insects should be just about completely removed from this field if ARSCL VAP processing has adequately done its job.

Note: This quantity is equivalent to the total area under the curve in Figure $4 b$ and is derived from measured Doppler spectra similar to the simulated spectrum in Figure $4 e$.

\subsubsection{ReflectivityBestEstimate}

Radar reflectivity (0th Moment) in dBZe from all heights, which our processing has indicated as containing hydrometeors. Regions that appear to contain insects, as well as hydrometeors, are contained in this field.

Note: This quantity is equivalent to the total area under the curve in Figure $4 b$ and is derived from measured Doppler spectra similar to the simulated spectrum in Figure $4 e$.

\subsubsection{MeanDopplerVelocity}

The mean Doppler velocity (1st Moment) generated from the spectra acquired over a period of about $10 \mathrm{~s}$.

Note: This quantity is equivalent to the average cross section weighted velocity of the curve in Figure $4 b$ and is derived from measured Doppler spectra similar to the simulated spectrum in Figure $4 e$.

\subsubsection{SpectralWidth}

The mean Doppler width (2nd Moment) generated from the spectra acquired over a period of about $10 \mathrm{~s}$.

Note: This quantity is equivalent to 2 times the standard deviation of the cross section weighted velocity about MeanDopplerVelocity, i.e., 2 times the standard deviation of the cross section versus velocity curve in Figure $4 b$, and is derived from measured Doppler spectra similar to the simulated spectrum in Figure $4 e$. 


\subsubsection{RadarFirstTop}

The height of the top of the lowest layer of significant returns in the Reflectivity field. This height corresponds either to the cloud top of the lowest cloud layer, if there is no clutter present near cloud top, or to the top of the clutter layer in those cases where the clutter layer extends above cloud top or occurs in clear sky. We originally used this variable in clutter tests, but now it plays no crucial role in the ARSCL VAP processing.

\subsubsection{Modeld}

The final set of reflectivities, mean Doppler velocities, and Doppler spectral widths are generated from 4 different modes. This variable flags which modes are contributing to the 3 fields as a function of time and height. Values of 1 (Mode 1) will not be seen much until after September 15, 1997. Values of 2 (Mode 2) are the cirrus mode. Values of 3 (Mode 3) are the general mode and should be the predominant color in most cases. Values of 4 (Mode 4) occur at times and heights where the hydrometeors have significant fall speeds and reflectivities, as might occur during precipitation.

\subsubsection{SignaltoNoiseRatio}

The signal-to-noise ratio in $\mathrm{dB}$ of the signal power returns to the radar receiver noise. As the signal-to-noise ratio increases, the Doppler moments become more reliable.

\subsubsection{CloudBasePrecipitation}

When the MWR wet window flag or the surface meteorology station rain gauge indicate precipitation at the surface, this flag is set to 1 . If all the laser-based cloud base height systems fail at this time, then a value of 0 is assigned to the cloud base height.

\subsubsection{CloudBaseCeilometerStd}

The cloud base height reported by the proprietary algorithms running on either the Belfort or Vaisala system.

\subsubsection{CloudBaseCeilometerCloth}

The cloud base height reported by the Clothiaux et al. (1998) cloud detection algorithm when it is applied to the backscatter profiles reported by the Belfort or Vaisala system. This algorithm has not yet been implemented.

\subsubsection{CloudBaseMpIScott}

The cloud base height reported by the Scott and Spinhirne algorithm when it is applied to the backscattered photon count profiles generated by the MPL. 


\subsubsection{CloudBaseMplCamp}

The cloud base height reported by the Campbell et al. (1998) algorithm when it is applied to the backscattered photon count profiles generated by the MPL.

\subsubsection{CloudBaseMpICloth}

The cloud base height reported by the Clothiaux et al. (1998) algorithm when it is applied to the backscattered photon count profiles generated by the MPL.

\subsubsection{CloudBaseBestEstimate}

Analyzing all the laser-derived cloud base heights together, we arrive at our best-estimate of the cloud base height. See Clothiaux et al. (2000) for a description of the current approach for doing this analysis.

\subsubsection{CloudMaskMpICamp}

Time-height cloud mask produced by the Campbell et al. (1998) cloud detection algorithm.

\subsubsection{CloudMaskMpICloth}

Time-height cloud mask produced by the Clothiaux et al. (1998) cloud detection algorithm.

\subsubsection{CloudLayerBottomHeightMpICamp}

The bottom height of each hydrometeor layer detected by the combined MMCR and Campbell et al. (1998) MPL algorithm cloud masks. More work may need to be done here, so be very careful with this variable.

\subsubsection{CloudLayerBottomHeightMplCloth}

The bottom height of each hydrometeor layer detected by the combined MMCR and Clothiaux et al. (1998) MPL algorithm cloud masks. More work may need to be done here, so be very careful with this variable.

\subsubsection{CloudLayerTopHeightMpICamp}

The top height of each hydrometeor layer detected by the combined MMCR and Campbell et al. (1998) MPL algorithm cloud masks. More work may need to be done here, so be very careful with this variable. 


\subsubsection{CloudLayerTopHeightMpICloth}

The top height of each hydrometeor layer detected by the combined MMCR and Clothiaux et al. (1998) MPL algorithm cloud masks. More work may need to be done here, so be very careful with this variable.

\subsubsection{5 qc_RadarArtifacts}

Each mode, with the exception of the current Mode 4, may have artifacts in the data. If some of these artifacts enter the final merged reflectivity field, then we flag the data as containing problems. In the current configuration of the ARM MMCRs, this should never be a problem. It will be a problem in the data that were collected before mid-October 1999 when the original set of modes were still in use.

\subsubsection{6 qc_ReflectivityClutterFlag}

Values of 0 indicate no significant power detection. Values of 1 (Flag Value 1) indicate a hydrometeor detection with no significant clutter (e.g., insect) contamination. Values of 2 indicate that both hydrometeors and insects are potentially contributing to the significant power returns to the radar. Under these conditions, the cloud top height is uncertain; that is, the top of the Value 2 flagged region may not be true cloud top height. However, cloud top height falls within the Value 2 region. Values of 3 (Flag Value 3) indicate regions where our processing says all of the significant power returns come from insects and other clutter. Finally, values of 10 (Flag Value 10) indicate that no data are present at this time for this range bin.

\subsubsection{7 qc_CloudLayerTopHeightMplCamp}

A value indicating whether or not the top height of each cloud layer detected by the combined MMCR and Campbell et al. (1998) MPL algorithm cloud masks represents the true cloud top. This variable must be set by an analysis of the MPL data, since there is no way to conclusively establish cloud top from radar returns. More work may need to be done here, so be very careful with this variable.

\subsubsection{8 qc_CloudLayerTopHeightMplCloth}

A value indicating whether or not the top height of each cloud layer detected by the combined MMCR and Clothiaux et al. (1998) MPL algorithm cloud masks represents the true cloud top. This variable must be set by an analysis of the MPL data, since there is no way to conclusively establish cloud top from radar returns. More work may need to be done here, so be very careful with this variable.

\subsubsection{9 qc_BeamAttenuationMpICamp}

Variable indicating what happened to the laser beam: data do not exist (-9), laser beam is blocked $(-2)$, laser beam is attenuated (-1), no cloud is detected by the beam (0), and the beam completely penetrated a cloud layer (1). 


\subsubsection{0 qc_BeamAttenuationMpICloth}

An experimental variable composed of the base 10 logarithm of signal power above cloud to clear-sky signal power at the same height. The hope is that this variable can be used to assess the degree of lidar attenuation through a cloud.

\subsection{Datastream arsclbnd1cloth}

To reduce the size of the datastream carrying information about the hydrometeor layer boundaries, the boundary fields in arscllcloth are extracted and placed in this datastream, together with the best-estimate cloud base height.

\subsection{1 base_time}

ARM standard time of the first record in seconds from the beginning of 1970.

\subsection{2 time_offset}

ARM standard time of the number of seconds from the base_time.

\subsubsection{CloudBaseBestEstimate}

Analyzing all the laser-derived cloud base heights together, we arrive at our best-estimate of the cloud base height. See Clothiaux et al. (2000) for a description of the current approach for doing this analysis.

\subsubsection{CloudLayerBottomHeightMpICamp}

The bottom height of each hydrometeor layer detected by the combined MMCR and Campbell et al. (1998) MPL algorithm cloud masks. More work may need to be done here, so be very careful with this variable.

\subsubsection{CloudLayerBottomHeightMplCloth}

The bottom height of each hydrometeor layer detected by the combined MMCR and Clothiaux et al. (1998) MPL algorithm cloud masks. More work may need to be done here, so be very careful with this variable.

\subsubsection{CloudLayerTopHeightMplCamp}

The top height of each hydrometeor layer detected by the combined MMCR and Campbell et al. (1998) MPL algorithm cloud masks. More work may need to be done here, so be very careful with this variable. 


\subsubsection{CloudLayerTopHeightMpICloth}

The top height of each hydrometeor layer detected by the combined MMCR and Campbell et al. (1998) MPL algorithm cloud masks. More work may need to be done here, so be very careful with this variable.

\subsection{8 qc_CloudLayerTopHeightMplCamp}

A value indicating whether or not the top height of each cloud layer detected by the combined MMCR and Campbell et al. (1998) MPL algorithm cloud masks represents the true cloud top. This variable must be set by an analysis of the MPL data, since there is no way to conclusively establish cloud top from radar returns. More work may need to be done here, so be very careful with this variable.

\subsection{9 qc_CloudLayerTopHeightMpICloth}

A value indicating whether or not the top height of each cloud layer detected by the combined MMCR and Clothiaux et al. (1998) MPL algorithm cloud masks represents the true cloud top. This variable must be set by an analysis of the MPL data, since there is no way to conclusively establish cloud top from radar returns. I do not believe the final set of algorithms have been developed yet.

\subsection{Datastream arsclcbh1cloth}

To reduce the size of the datastream carrying information about cloud base heights, the cloud base height fields in arscllcloth are extracted and placed in this datastream.

\subsection{1 base_time}

ARM standard time of the first record in seconds from the beginning of 1970.

\subsection{2 time_offset}

ARM standard time of the number of seconds from the base_time.

\subsubsection{CloudBasePrecipitation}

When the MWR wet window flag or the surface meteorology station rain gauge indicate precipitation at the surface, this flag is set to 1 . If all of the laser-based cloud base height systems fail at this time, then a value of 0 is assigned to the cloud base height.

\subsubsection{CloudBaseCeilometerStd}

The cloud base height reported by the proprietary algorithms running on either the Belfort or Vaisala system. 


\subsubsection{CloudBaseCeilometerCloth}

The cloud base height reported by the Clothiaux et al. (1998) cloud detection algorithm when it is applied to the backscatter profiles reported by the Belfort or Vaisala system. This algorithm has not yet been implemented.

\subsubsection{CloudBaseMpIScott}

The cloud base height reported by the Scott and Spinhirne algorithm when it is applied to the backscattered photon count profiles generated by the MPL.

\subsubsection{CloudBaseMpICamp}

The cloud base height reported by the Campbell et al. (1998) algorithm when it is applied to the backscattered photon count profiles generated by the MPL.

\subsubsection{CloudBaseMplCloth}

The cloud base height reported by the Clothiaux et al. (1998) algorithm when it is applied to the backscattered photon count profiles generated by the MPL.

\subsection{Datastream mmcrmode_ $\mathbf{v}$}

For studies of cloud climatologies, cloud existence, cloud location, etc., we expect the arscl1cloth datastream to be the most relevant one. However, as the MMCRs improve in temporal resolution, time

series analysis of the individual mode data may become important. The mmcrmode_v__ datastream was developed with time-series applications in mind. It contains the raw MMCR reflectivity data as it were originally collected by the MMCR. To aid in the interpration of these data, the laser-derived cloud base height information is moved to the mode data grid, together with the arscllcloth qc flags. Therefore, untouched data are available in these files, together with a series of flags and laser data that should aid in interpretation of these data.

\subsection{1 base_time}

ARM standard time of the first record in seconds from the beginning of 1970.

\subsection{2 time_offset}

ARM standard time of the number of seconds from the base_time.

\subsubsection{Heights}

A vector containing the height of the center of each MMCR mode range bin. 


\subsubsection{Reflectivity}

Radar reflectivity (0th Moment) in dBZe for all times and heights of the mode that the "Cloud Mask Code" algorithm has indicated as containing a significant power return, including power returns from insects and other clutter sources.

Note: This quantity is equivalent to the total area under the curve in Figure $4 b$ and is derived from measured Doppler spectra similar to the simulated spectrum in Figure $4 e$.

\subsubsection{MeanDopplerVelocity}

The mean Doppler velocity (1st Moment) generated from the mode spectra, which are acquired over a period of about $10 \mathrm{~s}$.

Note: This quantity is equivalent to the average cross section weighted velocity of the curve in Figure $4 b$ and is derived from measured Doppler spectra similar to the simulated spectrum in Figure 4e.

\subsubsection{SpectralWidth}

The mean Doppler width (2nd Moment) generated from the mode spectra, which are acquired over a period of about $10 \mathrm{~s}$.

Note: This quantity is equivalent to $\mathbf{2}$ times the standard deviation of the cross section weighted velocity about MeanDopplerVelocity, i.e., 2 times the standard deviation of the cross section versus velocity curve in Figure $4 b$, and is derived from measured Doppler spectra similar to the simulated spectrum in Figure $4 e$.

\subsubsection{SignaltoNoiseRatio}

The signal-to-noise ratio in $\mathrm{dB}$ of the signal power returns to the radar receiver noise. As the signal-to-noise ratio increases, the Doppler moments become more reliable.

\subsubsection{CloudBasePrecipitation}

When the MWR wet window flag or the surface meteorology station rain gauge indicate precipitation at the surface, this flag is set to 1 . If all of the laser-based cloud base height systems fail at this time, then a value of 0 is assigned to the cloud base height.

\subsubsection{CloudBaseCeilometerStd}

The cloud base height reported by the proprietary algorithms running on either the Belfort or Vaisala system. 


\subsubsection{CloudBaseCeilometerCloth}

The cloud base height reported by the Clothiaux et al. (1998) cloud detection algorithm when it is applied to the backscatter profiles reported by the Belfort or Vaisala system. This algorithm has not yet been implemented.

\subsubsection{CloudBaseMplScott}

The cloud base height reported by the Scott and Spinhirne algorithm when it is applied to the backscattered photon count profiles generated by the MPL.

\subsubsection{CloudBaseMpICamp}

The cloud base height reported by the Campbell et al. (1998) algorithm when it is applied to the backscattered photon count profiles generated by the MPL.

\subsubsection{CloudBaseMpICloth}

The cloud base height reported by the Clothiaux et al. (1998) algorithm when it is applied to the backscattered photon count profiles generated by the MPL.

\subsubsection{CloudBaseBestEstimate}

Analyzing all the laser-derived cloud base heights together, we arrive at our best-estimate of the cloud base height. See Clothiaux et al. (2000) for a description of the current approach for doing this analysis.

\subsubsection{5 qc_RadarArtifacts}

Unlike the arscllcloth datastream, where all the radar artifacts have been removed, the data for each individual mode may have one of a number of artifacts in the data. This qc flag indicates the problems in the data.

\subsubsection{6 qc_ReflectivityClutterFlag}

Values of 0 indicate no significant power detection. Values of 1 (Flag Value 1) indicate a hydrometeor detection with no significant clutter (e.g., insect) contamination. Values of 2 indicate that both hydrometeors and insects are potentially contributing to the significant power returns to the radar. Under these conditions, the cloud top height is uncertain; that is, the top of the Value 2 flagged region may not be true cloud top height. However, cloud top height falls within the Value 2 region. Values of 3 (Flag Value 3) indicate regions where our processing says all of the significant power returns come from insects and other clutter. Values of 4 (Flag Value 4) indicate that there is a significant detection in the arscllcloth product at this range and time but not in the mode data. Finally, values of 10 (Flag Value 10) indicate that no data is present at this time for this range bin. 


\section{ARSCL VAP: Product Images}

The ARSCL product images listed below are available through the ARM Scientific Application Quick-Look (SAQL) database.

\subsection{Datastream blcprof lidar_amplitude}

In order to inspect the blcprof backscattered power returns in a form identical to the MMCR data, we take the logarithm to the base ten of the lidar_amplitude variable to create the parameter LogPowerReturn. We then plot LogPowerReturn on a timeheight axis that spans a day and ranges from the surface to $3 \mathrm{~km}$ above ground level (AGL). An image is made of these data and labeled with the name lidar_amplitude. The netCDF file is subsequently discarded.

\subsection{Datastream blcprof clearcloud}

Image of blcprof cloud base height, together with clear/cloud flags.

\subsection{Datastream vceil25k backscatterbase}

To inspect the vceil $25 \mathrm{k}$ backscattered power returns in a form identical to the MMCR data, we take the logarithm to the base ten of the power return variable to create the parameter LogPowerReturn. We then plot LogPowerReturn on a time-height axis that spans a day and ranges from the surface to $3 \mathrm{~km}$ AGL. An image is made of these data and labeled with the name backscatterbase. The netCDF file is subsequently discarded.

\subsection{Datastream vceil25k backscatternobase}

Identical to the previous image, except vceil25k cloud base height is also included.

\subsection{Datastream vceil25k clearcloud}

Image of vceil25k cloud base height, together with clear/cloud flags.

\subsection{Datastream mwrlos vapliqwetwindowxy}

Image of mwrlos water vapor, liquid water, and wet window flag.

\subsection{Datastream mwrlos clearcloud}

Image of mwrlos wet window flag, together with clear/cloud flags.

\subsection{Datastream mpl background}

Image of solar radiation scattered into MPL receiver field of view. 


\subsection{Datastream mpl backscatter}

In order to inspect the MPL backscattered power returns in a form identical to the MMCR data, we take the logarithm to the base ten of the power return variable to create the parameter LogPowerReturn. We then plot LogPowerReturn on a time-height axis that spans a day and ranges from the surface to $20 \mathrm{~km}$ AGL. An image is made of these data and labeled with the name backscatter. The netCDF file is subsequently discarded.

\subsection{Datastream mpl backscatterBL}

Identical to the previous image, the image is up to only $3 \mathrm{~km}$. Note that BL stands for boundary layer.

\subsection{Datastream $\mathrm{mpl}$ clearcloud}

Image of MPL cloud base height from the Clothiaux et al. (1998) algorithm, together with clear/cloud flags.

\subsection{Datastream mplcbh1scott clearcloud}

Image of MPL cloud base height from the Scott et al. algorithm (Clothiaux et al. 1998), together with clear/cloud flags.

\subsection{Datastream mplnor1camp backscatter}

Image of MPL normalized backscatter from the Campbell et al. algorithm (Campbell et al. 1998).

\subsection{Datastream mplnor1camp cloudmask}

Image of MPL cloud mask from the Campbell et al. algorithm (Campbell et al. 1998).

\subsection{Datastream mplnor1camp clearcloud}

Image of MPL cloud base height from the Campbell et al. algorithm (Campbell et al. 1998), together with clear/cloud flags.

\subsection{Datastream mplsmask1cloth SigniMaskMpICloth}

See Section 5.1.

\subsection{Datastream mplcmask1cloth CloudMaskMpICloth}

See Section 5.2. 


\subsection{Datastream arscl1cloth CloudMaskMplCamp}

See Section 5.3.

\subsection{Datastream arscl1cloth CloudMaskMpICloth}

See Section 5.3.

\subsection{Datastream arscl1cloth LaserCloudBases}

See Section 5.3.

\subsection{Datastream arscl1cloth Reflectivity}

See Section 5.3.

\subsection{Datastream arscl1cloth ReflectivityNoClutter}

See Section 5.3.

\subsection{Datastream arscl1cloth ReflectivityBestEstimate}

See Section 5.3.

\subsection{Datastream arscl1cloth MeanDopplerVelocity}

See Section 5.3.

\subsection{Datastream arscl1cloth SpectralWidth}

See Section 5.3.

\subsection{Datastream arscl1cloth Modeld}

See Section 5.3.

\subsection{Datastream arscl1cloth SignaltoNoiseRatio}

See Section 5.3.

\subsection{Datastream arscl1cloth qc_RadarArtifacts}

See Section 5.3. 


\subsection{Datastream arscl1cloth qc_ReflectivityClutterFlag}

See Section 5.3.

\subsection{Datastream arsclbnd1cloth CloudLayerHeightsCloth}

See Section 5.4.

\subsection{Datastream arsclbnd1cloth qc_CloudLayerHeightsCloth}

See Section 5.4.

6.32 Datastream arsclcbh1cloth LaserCloudBases

See Section 5.5.

\subsection{Datastream mmcrmode__v__ 1cloth Reflectivity}

See Section 5.6.

\subsection{Datastream mmcrmode_ $v \_$1cloth ReflectivityBL}

See Section 5.6.

\subsection{Datastream mmcrmode_ $\mathbf{v} \_$1cloth qc_RadarArtifacts}

See Section 5.6.

\subsection{Datastream mmcrmode__ $\mathbf{v}$ _ 1cloth qc_ReflectivityClutterFlag}

See Section 5.6.

\subsection{Datastream mmcrmode_v__ 1cloth qc_ReflectivityClutterFlagBL}

See Section 5.6. 


\section{ARSCL VAP: Data Quality Issues}

\subsection{Quality Control (QC) Flags}

\section{Date: 09 March 2001}

The most useful quality control (qc) flag in the arscllcloth datastream is qc_ReflectivityClutterFlag. This flag, coupled with the values of Reflectivity, ReflectivityNoClutter, and ReflectivityBestEstimate, can be used to sort the data in the arscllcloth datastream files. The logic is as follows:

1. If qc_ReflectivityClutterFlag equals 10, no data exist for this period.

2. If qc_ReflectivityClutterFlag does not equal 10 but the relevant reflectivity value is either less than -100 or greater than 30 (after dividing the file value by 100), data exist but there is no significant detection (i.e., no cloud) for this time period.

3. If qc_ReflectivityClutterFlag does not equal 10 and the relevant reflectivity value is either greater than -100 or less than 30 (after dividing the file value by 100), data exist, there is a significant detection for this time period, and the value of qc_ReflectivityClutterFlag provides our guess as to the source of the power return.

\subsection{MMCR Calibration}

\section{Date: 09 March 2001}

The absolute calibration of all four (i.e., SGP, TWP-Manus, TWP-Nauru, and NSA) ARM MMCRs is thought to be good to at least $1 \mathrm{~dB}$. Several calibration experiments with the ARM SGP MMCR support this estimate of accuracy. While the remaining three MMCRs have not been directly calibrated, the calibration procedures for these three radars are identical to the procedures applied to the ARM SGP MMCR. These procedures include transmit power monitoring and incorporation of the measurements into the reflectivity calculations, as well as automatic receiver calibration with a calibrated noise source.

\section{Date: 09 March 2001}

In the near-field of the MMCRs, i.e., below about $1 \mathrm{~km}$, a correction must be made to the reflectivities. At the DOE ARM Cloud Working Group Meeting at the end of January 2001, NOAA ETL presented results from Steve Sekelsky demonstrating that the near-field correction applied to the ARM MMCR data is off by about 2 to $3 \mathrm{~dB}$ near the surface and drops to a few tenths of a $\mathrm{dB}$ off approximately $1 \mathrm{~km}$ above the radar. A correction factor for this problem is being developed and will be made available as soon as possible. When the MMCR data are next reprocessed, this correction will be incorporated into the data. 


\subsection{SGP Belfort Laser Ceilometer Height Offsets}

\section{Date: 09 March 2001}

From the beginning of its data record until 3 February 2000, the Belfort laser ceilometer heights are too high by $122 \mathrm{~m}$. On 4 February 2000, the height offset was finally removed from the Belfort ceilometer raw data and, consequently, from the ARSCL VAP products. When the BLC raw data files and ARSCL VAP products are reprocessed, this height offset will finally be removed from ARM data.

\subsection{NSA Micropulse Lidar Height Offsets}

\section{Date: 09 March 2001}

When the NSA MPL was brought back to life on 16 June 1999, a height offset of $30 \mathrm{~m}$ appeared in its backscatter profiles. That is, the MPL heights are $30 \mathrm{~m}$ too high starting on this date. This problem continued until Day Month Year when it was finally removed. Raw MPL data during the period of height offset will have to be reprocessed at some point, as well as the ARSCL VAP data for this period.

\section{ARSCL VAP: Retrieving Data from the ARM Archive}

Note that ARSCL VAP products are from the SGP C1, NSA C1, TWP C1 (Manus), and TWP C2 (Nauru) sites. Furthermore, all ARSCL VAP products are c1 level data files. So, ARSCL VAP file names can be obtained by taking the datastream names in Section 5 and prefixing them with the site and suffixing them with either C1.c1 or C2.c1. For example, datastream arscl1cloth files from the SGP central facility are labeled sgparscllclothC1.c1 and those from TWP-Nauru are labeled twparscl1clothC2.c1.

All DOE ARM Program data are available from the DOE ARM data archive. One way to access ARM data is by doing the following:

1. Use the Internet to access http://www.archive.arm.gov/cgi-bin/arm-archive.

2. "Login Archive" with appropriate "Username."

3. Choose the "Request data using Query Interface" option.

4. Choose the "Data Streams" option and then click on the appropriate DOE ARM site (i. e., SGP, NSA, TWP) box.

5. From the "Production Data Streams" column listing datastreams.

6. Select sgparscl1clothC1.c1, sgparsclbnd1clothC1.c1, etc., if the SGP site was chosen. 


\section{Select the "Dates," both "Starting" and "Ending," and then click on "Create Order."}

8. Click the "Place Order," "Return," and "Logoff Archive" buttons, and then wait for your automated e-mail message from the ARM archive telling you where to pick up your data.

\section{Conclusions}

The primary goal of the ARM 35-GHz radars, or MMCRs, is to provide a continuous record of clouds and their properties in the vertical column above the ARM sites. The ARM SGP radar has been operational since November 1996; it is currently collecting data using the sequence of four modes described above (Moran et al. 1998; Clothiaux et al. 1999). The NSA, TWP-Manus, and TWP-Nauru radars began operation in March 1998, June 1999, and November 1998, respectively, and they are currently operating in a similar fashion to the SGP radar. Slight changes to the parameters of the TWP and NSA MMCR operational modes were made because of differences in cloud properties with respect to the ARM SGP site. Combining data from the four modes leads to an accurate description of the cloud coverage in the vertical column above the radar during all conditions, except when insects are present and for rain when attenuation of the 35-GHz beam becomes significant (Clothiaux et al. 2000). Detecting the presence of clouds and cloud boundaries is the first step in ascertaining the macrophysical and microphysical properties of the clouds in the vertical column above the radar. Once the presence of clouds has been ascertained through algorithms such as those developed by Uttal et al. (1993) or Clothiaux et al. (1995), the next important step is retrieving the microphysical properties of the cloud particles, which is the subject of current ongoing research.

Each mode of the MMCRs currently has a temporal resolution of approximately $9 \mathrm{~s}$. The temporal resolution can be shortened, but at the cost of lower sensitivity. The efficiency of the radar signal processor currently ranges from $4 \%$ to $31 \%$ for SGP Modes 4 through 1, as depicted in Figure 3; that is, the computers can process only a small portion of the data that the radar can generate. With improvements to the radar signal processing systems, the temporal resolution of each mode can approach approximately $0.45 \mathrm{~s}$ without a loss of sensitivity.

Although power density spectra (e.g., Figure 4e) are produced routinely by the MMCRs, they can be saved at only selected times because of the large amount of memory they require. The first three moments derived from the spectra (i.e., the reflectivity, the mean velocity, and the standard deviation of the velocity) are saved at full temporal resolution. Fortunately, Moran et al. (1998) configured the radar to be run remotely, so the exact sequence of modes running the radar, including whether or not the power density spectra are saved, can be changed quickly in order to capture interesting cloud events, using more appropriate modes.

Images of the data from the ARM radars are available at a number of Web sites (see the ARM home page). These graphic images range from quick-look images of the data that are up-to-date (no more than two hours behind real time), to archives of data over the past two weeks, to archives of the data over an 
entire experimental period (except for the past two months). Original radar data will eventually be available from the ARM data archive in Oak Ridge, Tennessee. Eventually, all the ARSCL VAP datastreams will be found at the ARM archive. In the interim, these data can be obtained by contacting any of the contributors to this technical report.

\section{Acknowledgements}

Support for this research was funded by the Environmental Sciences Division of the U.S. Department of Energy (under Grant DE-FG02-90ER61071) and Battelle Pacific Northwest National Laboratory (Subcontract 091572-A-Cal).

\section{References}

Atlas, D., S. Y. Matrosov, A. J. Heymsfield, M.D. Chou, and D. B. Wolff, 1995: Radar and radiation properties of ice clouds. J. Appl. Meteor., 34, 2329-2345.

Battan, L. J., 1973: Radar Observation of the Atmosphere. University of Chicago Press, Chicago, Illinois.

Campbell, J. R., D. L. Hlavka, J. D. Spinhirne, V. S. Scott, and D. D. Turner, 1998: Operational processing and cloud boundary detection from micro pulse lidar data. Preprints of the Nineteenth International Laser Radar Conference, Ed. UN Singh, NASA CP-1998-207671/PT1, Annapolis, Maryland, July, pp 119-122.

Clothiaux, E. E., M. A. Miller, B. A. Albrecht, T. P. Ackerman, J. Verlinde, D. M. Babb, R. M. Peters, and W. J. Syrett, 1995: An evaluation of a 94-GHz radar for remote sensing of cloud properties. J. Atmos. Oceanic Technol., 12, 201-229.

Clothiaux, E. E., G. G. Mace, T. P. Ackerman, T. J. Kane, J. D. Spinhirne, and V. S. Scott, 1998: An automated algorithm for detection of hydrometeor returns in micro pulse lidar data. J. Atmos. and Oceanic Technol., 15, 1035-1042:

Clothiaux, E. E., K. P. Moran, B. E. Martner, T. P. Ackerman, G. G. Mace, T. Uttal, J. H. Mather, K. B. Widener, M. A. Miller, and D. J. Rodriguez, 1999: The Atmospheric Radiation Measurement program cloud radars: Operational modes. J. Atmos. Oceanic Technol., 16, 819-827.

Clothiaux, E. E., T. P. Ackerman, G. G. Mace, K. P. Moran, R. T. Marchand, M. A. Miller, and B. E. Martner, 2000: Objective determination of cloud heights and radar reflectivities using a combination of active remote sensors at the ARM CART sites. J. Appl. Meteor., 39, 645-665.

Doviak, R. J., and D. S. Zrnic, 1984: Doppler Radar and Weather Observations. Academic Press, San Diego, California. 
Doviak, R. J., and D. S. Zrnic, 1993: Doppler Radar and Weather Observations. Second Edition. Academic Press, San Diego, California.

Fox, N. L, and A. J. Illingworth, 1997: The potential of a spaceborne cloud radar for the detection of stratocumulus clouds. J. Appl. Meteor., 36, 676-687.

Frisch, A. S., C. W. Fairall, and J. B. Snider, 1995: Measurement of stratus cloud and drizzle parameters in ASTEX with a K,-band Doppler radar and a microwave radiometer. J. Atmos. Sci., 52, 2788-2799.

Heymsfield, A. J., L. M. Miloshevich, A. Slingo, K. Sassen, and D. O’C. Starr, 1991: An observational and theoretical study of highly supercooled altocumulus. J. Atmos. Sci., 48, 923-945.

Moran, K. P., B. E. Martner, D. C. Welsh, D. A. Merritt, M. J. Post, T. Uttal, and R. G. Strauch, 1997: ARM's cloud profiling radar. 28th Conference on Radar Meteorology, Austin, Texas, Am. Meteor. Soc., 296-297.

Moran, K. P., B. E. Martner, M. J. Post, R. A. Kropfli, D. C. Welsh and K. B. Widener, 1998: An unattended cloud-profiling radar for use in climate research. Bull. Amer. Meteor. Soc., 79, 443-455.

Noonkester, V. R., 1984: Droplet spectra observed in marine stratus cloud layers. J. Atmos. Sci., 41, 829-845.

Rogers, R. R., D. Baumgardner, S. A. Ethier, D. A. Carter, and W. L. Ecklund, 1993: Comparison of raindrop size distributions measured by radar wind profiler and by airplane. J. Appl. Meteor., 32, 694-699.

Sauvageot, H., 1992: Radar Meteorology. Artech House, Inc., Norwood, Massachussetts.

Schmidt, G., R. Ruster, and P. Czechowsky, 1979: Complementary code and digital filtering for detection of weak VHF radar signals from the mesosphere. IEEE Trans. Geosci. Electron., 17, 154-161.

Skolnik, M., 1990: Radar Handbook. Second Edition. McGraw Hill, Inc., New York.

Ulaby, R. T., R. K. Moore, and A. K. Fung, 1982: Microwave Remote Sensing Active and Passive, Vol. II: Radar Remote Sensing and Surface Scattering and Emission Theory. Artech House, Inc., Norwood, Massachussetts.

Uttal, T., L. I. Church, B. E. Martner, and J. S. Gibson, 1993: CLDSTATS: A cloud boundary detection algorithm for vertically pointing radar data. NOAA Tech. Memorandum ERL WPL-233, Environmental Technology Laboratory, Boulder, Colorado. 


\section{Appendix A1 - ARSCL VAP Input Datastreams}

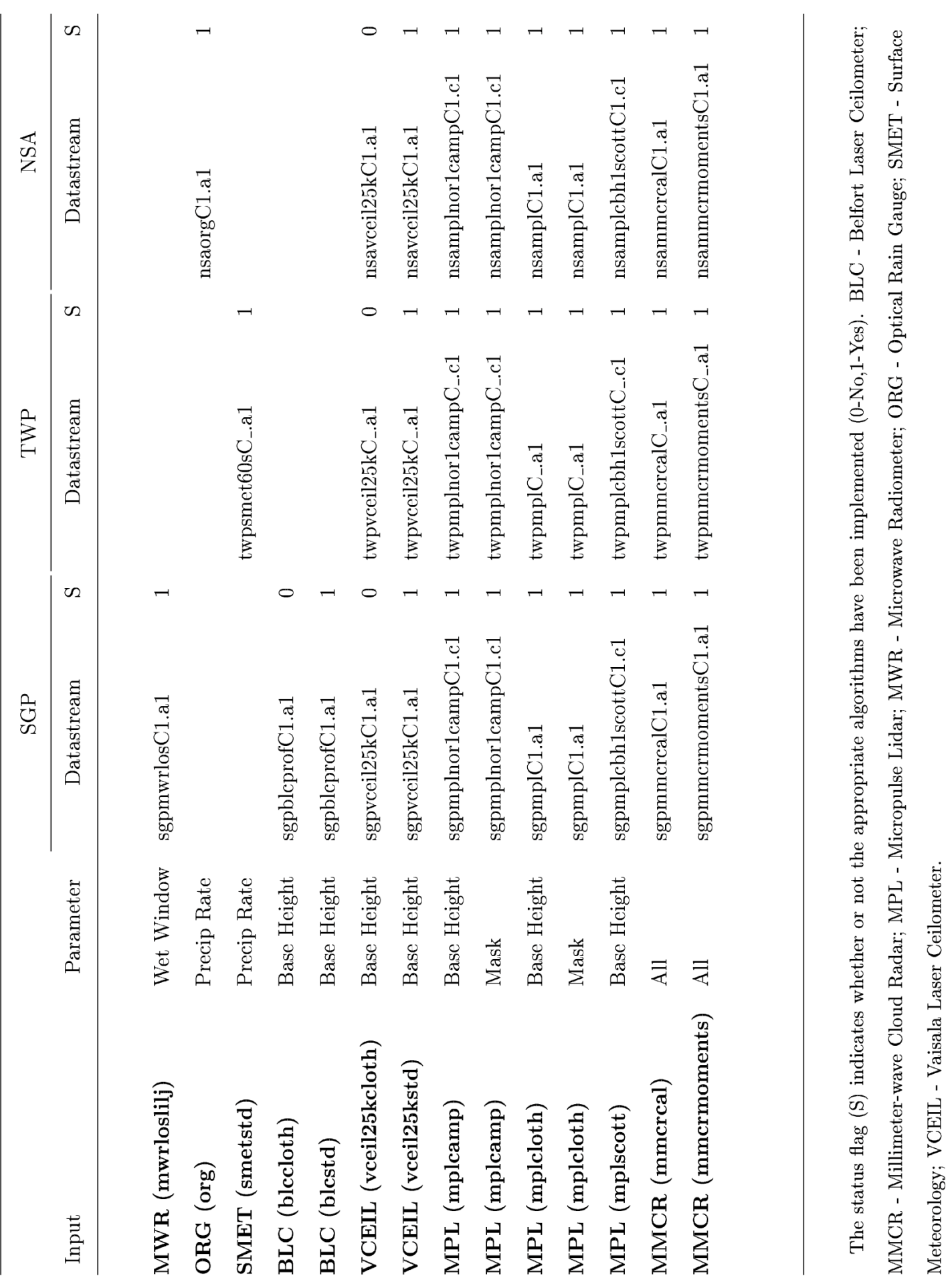

Figure A1. Datastream inputs to the ARSCL VAP. 


\section{Appendix A2 - ARSCL VAP Flow Chart}

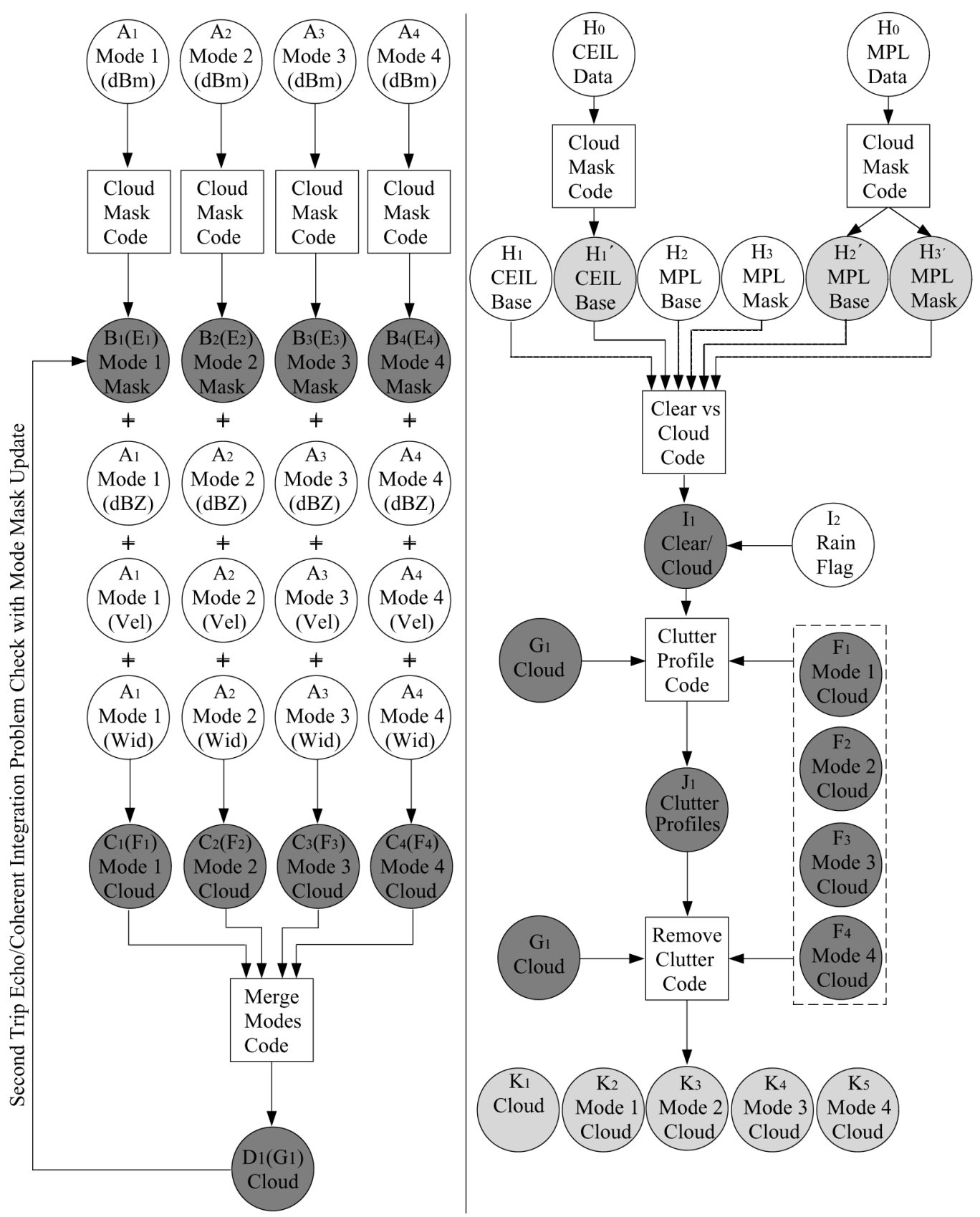

Figure A2. Flow chart of the ARSCL VAP. Open circles represent one of the input datastreams, open squares represent an algorithmic process that implements some of the ARSCL VAP functionality, dark gray circles represent intermediate files kept either in internal memory as arrays or in netCDF files on a hard disk, and light gray circles represent the netCDF output files generated by the ARSCL VAP. 


\title{
Appendix A3 - ARSCL VAP Product Datastreams
}

\author{
DATASTREAM: mplsmask1cloth
}

\section{CONTACT:}

DIMENSIONS:

1) time $=$ UNLIMITED

2) nheights $=2001$

3) namelen $=64$

\section{VARIABLES:}

1) base_time:

long_name: Beginning Time of File

units: seconds since 1970-01-01 00:00:00 00:00

calendar_date: Year 0000 Month 00 Day 00 00:00:00

2) time_offset:

long_name: Time Offset from base_time

units: seconds

comment: none

3) Heights:

long_name: Height of Center of Each Range Bin

units: $\mathrm{m}$

comment: none

4) SigniMaskMplCloth:

long_name: MPL Cloth et al. Algorithm Significance Mask

units: unitless

comment: 0 No data, no retrieval, or no detection; 1 Significant detection from clearsky algorithm; 2 Significant detection from threshold step algorithm; 3 Significant detection from both algorithms

\section{COMMENTS:}


DATASTREAM: mplcmask1cloth

\section{CONTACT:}

\section{DIMENSIONS:}

1) time $=$ UNLIMITED

2) nheights $=2001$

3) namelen $=64$

\section{VARIABLES:}

1) base_time:

long_name: Beginning Time of File units: seconds since 1970-01-01 00:00:00 00:00

calendar_date: Year 0000 Month 00 Day 00 00:00:00

2) time_offset:

long_name: Time Offset from base_time units: seconds

comment: none

3) Heights:

long_name: Height of Center of Each Range Bin

units: $\mathrm{m}$

comment: none

4) CloudMaskMplCloth:

long_name: MPL Cloth et al. Algorithm Cloud Mask

units: unitless

comment: -2 Beam Blocked; -1 Beam Attenuated; 0 No Cloud (Clear); 1 Cloud Detected

5) qc_BeamAttenuationMplCloth:

long_name: MPL Cloth et al. Algorithm Beam Attenuation Assessment units: unitless

comment: Log10(Signal Power above Cloud/Clearsky Power above Cloud)

\section{COMMENTS:}


DATASTREAM: arscl1cloth

\section{CONTACT:}

\section{DIMENSIONS:}

1) time $=$ UNLIMITED

2) nheights $=512$

3) numlayers $=10$

\section{VARIABLES:}

1) base_time:

long_name: Beginning Time of File units: seconds since 1970-01-01 00:00:00 00:00

calendar_date: Year 0000 Month 00 Day 00 00:00:00

2) time_offset:

long_name: Time Offset from base_time

units: seconds

comment: none

3) Heights:

long_name: Height of Measured Value units: $\mathrm{m}$ AGL

comment: none

\section{4) Reflectivity:}

long_name: MMCR Reflectivity

units: dBZ (X100)

comment: Divide Reflectivity by 100 to get dBZ

5) ReflectivityNoClutter:

long_name: MMCR Reflectivity with Clutter Removed units: $\mathrm{dBZ}$ (X100)

comment: Divide ReflectivityNoClutter by 100 to get $\mathrm{dBZ}$

6) ReflectivityBestEstimate:

long_name: MMCR Best Estimate of Hydrometeor Reflectivity units: dBZ (X100)

comment: Divide ReflectivityBestEstimate by 100 to get $\mathrm{dBZ}$

7) MeanDopplerVelocity:

long_name: MMCR Mean Doppler Velocity

units: $\mathrm{m} / \mathrm{s}$ (X1000)

comment: Divide MeanDopplerVelocity by 1000 to get m/s

8) SpectralWidth:

long_name: MMCR Spectral Width

units: $\mathrm{m} / \mathrm{s}$ (X1000)

comment: Divide SpectralWidth by 1000 to get $\mathrm{m} / \mathrm{s}$

9) RadarFirstTop:

long_name: MMCR Top Height of Lowest Detected Layer before Clutter Removal units: $\mathrm{m}$ AGL

comment: -3 . Data do not exist, 0. No significant detection in column, \& 0. Top Height of Lowest Cloud/Clutter Layer

10) ModeId: 
long_name: MMCR Mode I.D.

units: unitless

comment: 0 No significant power return, 1-5 Valid modes, 10 Data do not exist

11) SignaltoNoiseRatio:

long_name: MMCR Signal-to-Noise Ratio

units: $\mathrm{dB}$ (X100)

comment: Divide SignaltoNoiseRatio by 100 to get $\mathrm{dB}$

12) CloudBasePrecipitation:

long_name: Microwave Radiometer Wet Window/Optical Rain Gauge Cloud Base Height units: $\mathrm{m}$ AGL

comment: -3 . Data do not exist, -2 . Data exist but no retrieval, -1 . Clear sky, $i=0$. Valid cloud base height

13) CloudBaseCeilometerStd:

long_name: BLC/VCEIL Standard Algorithm Cloud Base Height

units: $\mathrm{m}$ AGL

comment: -3 . Data do not exist, -2 . Data exist but no retrieval, -1 . Clear sky, $i=0$. Valid cloud base height

14) CloudBaseCeilometerCloth:

long_name: BLC/VCEIL Clothiaux et al. Algorithm Cloud Base Height

units: $\mathrm{m}$ AGL

comment: -3 . Data do not exist, -2 . Data exist but no retrieval, -1 . Clear sky, $i=0$. Valid cloud base height

15) CloudBaseMplScott:

long_name: MPL Scott Algorithm Cloud Base Height

units: $\mathrm{m}$ AGL

comment: -3 . Data do not exist, -2 . Data exist but no retrieval, -1 . Clear sky, $i=0$. Valid cloud base height

16) CloudBaseMplCamp:

long_name: MPL Campbell et al. Algorithm Cloud Base Height

units: $\mathrm{m}$ AGL

comment: -3 . Data do not exist, -2 . Data exist but no retrieval, -1 . Clear sky, $i=0$. Valid cloud base height

17) CloudBaseMplCloth:

long_name: MPL Clothiaux et al. Algorithm Cloud Base Height

units: $\mathrm{m}$ AGL

comment: -3 . Data do not exist, -2 . Data exist but no retrieval, -1 . Clear sky, $\dot{i}=0$. Valid cloud base height

18) CloudBaseBestEstimate:

long_name: LASER Cloud Base Height Best Estimate

units: $\mathrm{m}$ AGL

comment: -3 . Data do not exist, -2 . Data exist but no retrieval, -1 . Clear sky, $i=0$. Valid cloud base height

19) CloudMaskMplCamp:

long_name: MPL Campbell et al. Algorithm Cloud Mask Occurrence units: Percent $(\mathrm{x} 100)$

comment: -30000 Data do not exist, -20000 Beam blocked, -10000 Beam attenuated, 0 No cloud (clear), \& 0 Valid cloud percent occurrence (x 100) during time interval.

20) CloudMaskMplCloth:

long_name: MPL Clothiaux et al. Algorithm Cloud Mask Occurrence 
units: Percent(x100)

comment: -30000 Data do not exist, -20000 Beam blocked, -10000 Beam attenuated, 0 No cloud (clear), \& 0 Valid cloud percent occurrence (x 100) during time interval.

\section{1) CloudLayerBottomHeightMplCamp:}

long_name: Bottom Height of Hydrometeor Layer from Composite (MMCR/Campbell et al. MPL) Algorithms

units: $\mathrm{m}$ AGL

comment: none

22) CloudLayerBottomHeightMplCloth:

long_name: Bottom Height of Hydrometeor Layer from Composite (MMCR/Clothiaux et al. MPL) Algorithms

units: $\mathrm{m}$ AGL

comment: none

23) CloudLayerTopHeightMplCamp:

long_name: Top Height of Hydrometeor Layer from Composite (MMCR/Campbell et al. MPL) Algorithms

units: $\mathrm{m}$ AGL

comment: none

24) CloudLayerTopHeightMplCloth:

long_name: Top Height of Hydrometeor Layer from Composite (MMCR/Clothiaux et al. MPL) Algorithms

units: $\mathrm{m}$ AGL

comment: none

25) qc_RadarArtifacts:

long_name: MMCR Mode Quality Control Flags

units: unitless

comment: 0 No significant power return, 1 Significant, problem free data, 2 Second trip echo problems, 3 Coherent integration problems, 4 Second trip echo and coherent integration problems, 5 Pulse coding problems, 10 Data do not exist

26) qc_ReflectivityClutterFlag:

long_name: MMCR Reflectivity Clutter Flags

units: unitless

comment: 0 No significant power return, 1 Significant, problem free data, 2 Clutter and cloud contribution, 3 Clutter only contribution, 10 Data do not exist

27) qc_CloudLayerTopHeightMplCamp:

long_name: Value Indicating the Reliability of the Layer Top Height Using the Campbell et al. MPL Algorithm

units: unitless

comment: none

28) qc_CloudLayerTopHeightMplCloth:

long_name: Value Indicating the Reliability of the Layer Top Height Using the Clothiaux et al. MPL Algorithm

units: unitless

comment: none

29) qc_BeamAttenuationMplCamp:

long_name: MPL Campbell et al. Algorithm Beam Attenuation Assessment

units: unitless

comment: -9. Data do not exist, -2. Beam blocked, -1. Beam attenuated, 0. No cloud (clear), 1. Beam penetrated atmosphere 


\section{0) qc_BeamAttenuationMplCloth:}

long_name: MPL Cloth et al. Algorithm Beam Attenuation Assessment units: unitless

comment: -9. Data do not exist, Log10(Signal Power above Cloud/Estimated Clearsky Power above Cloud)

\section{COMMENTS:}

At each height and time, the MMCR reflectivity, velocity, width and signal-to-noise ratio always come from the same mode. The mode is indicated by ModeId. MeanDopplerVelocity, ModeId, qc_RadarArtifacts, qc_ReflectivityClutterFlag, SpectralWidth, and SignaltoNoiseRatio data are reported at all range gates for which there is a significant detection, including from clutter. The value of qc_ReflectivityClutterFlag indicates whether or not the signal is from clutter. Use the appropriate reflectivity fields (e.g., with clutter, with clutter removed, or best estimate) to filter the variables discussed in commentb. Missing (i.e., does not exist) data for a particular time period are indicated by a value of 10 for the ModeId, qc_RadarArtifacts, and qc_ReflectivityClutterFlag variables. The geophysical variables should contain a value of -32768 at these times. Note that -32768 is also used for the geophysical variables when there are no significant detections, in which case ModeId, qc_RadarArtifacts, and qc_ReflectivityClutterFlag are 0. 


\section{DATASTREAM: arsclbnd1cloth}

\section{CONTACT:}

\section{DIMENSIONS:}

1) time $=$ UNLIMITED

2) numlayers $=10$

\section{VARIABLES:}

1) base_time:

long_name: Beginning Time of File

units: seconds since 1970-01-01 00:00:00 00:00

calendar_date: Year 0000 Month 00 Day 00 00:00:00

2) time_offset:

long_name: Time Offset from base_time

units: seconds

comment: none

3) CloudBaseBestEstimate:

long_name: LASER Cloud Base Height Best Estimate units: $\mathrm{m}$ AGL

comment: -3 . Data do not exist, -2 . Data exist but no retrieval, -1 . Clear sky, $i=0$. Valid cloud base height

4) CloudLayerBottomHeightMplCamp:

long_name: Bottom Height of Hydrometeor Layer from Composite (MMCR/Campbell et al. MPL) Algorithms

units: $\mathrm{m}$ AGL

comment: none

5) CloudLayerBottomHeightMplCloth:

long_name: Bottom Height of Hydrometeor Layer from Composite (MMCR/Clothiaux et al. MPL) Algorithms

units: $\mathrm{m}$ AGL

comment: none

6) CloudLayer'TopHeightMplCamp:

long_name: Top Height of Hydrometeor Layer from Composite (MMCR/Campbell et al. MPL) Algorithms

units: $\mathrm{m}$ AGL

comment: none

7) CloudLayerTopHeightMplCloth:

long_name: Top Height of Hydrometeor Layer from Composite (MMCR/Clothiaux et al. MPL) Algorithms

units: $\mathrm{m}$ AGL

comment: none

8) qc_CloudLayerTopHeightMplCamp:

long_name: Value Indicating the Reliability of the Layer Top Height Using the Campbell et al. MPL Algorithm

units: unitless

comment: none

9) qc_CloudLayerTopHeightMplCloth: 
long_name: Value Indicating the Reliability of the Layer Top Height Using the Clothiaux et al. MPL Algorithm

units: unitless

comment: none

\section{COMMENTS:}

If all layer top heights are 0 , then the first bottom layer height of 0 means no cloud layer detected. If there is a single non-zero cloud top height and the corresponding bottom layer height is 0 , then the cloud layer bottom is at ground level. 


\section{DATASTREAM: arsclcbh1cloth}

\section{CONTACT:}

\section{DIMENSIONS:}

1) time $=$ UNLIMITED

\section{VARIABLES:}

1) base_time:

long_name: Beginning Time of File

units: seconds since 1970-01-01 00:00:00 00:00

calendar_date: Year 0000 Month 00 Day 00 00:00:00

2) time_offset:

long_name: Time Offset from base_time

units: seconds

comment: none

3) CloudBasePrecipitation:

long_name: Microwave Radiometer Wet Window/Optical Rain Gauge Cloud Base Height units: $\mathrm{m}$ AGL

comment: -3 . Data do not exist, -2 . Data exist but no retrieval, -1 . Clear sky, $i=0$. Valid cloud base height

4) CloudBaseCeilometerStd:

long_name: BLC/VCEIL Standard Algorithm Cloud Base Height

units: $\mathrm{m}$ AGL

comment: -3 . Data do not exist, -2 . Data exist but no retrieval, -1 . Clear sky, $i=0$. Valid cloud base height

5) CloudBaseCeilometerCloth:

long_name: BLC/VCEIL Clothiaux et al. Algorithm Cloud Base Height

units: $\mathrm{m}$ AGL

comment: -3 . Data do not exist, -2 . Data exist but no retrieval, -1 . Clear sky, $i=0$. Valid cloud base height

6) CloudBaseMplScott:

long_name: MPL Scott Algorithm Cloud Base Height

units: $\mathrm{m}$ AGL

comment: -3 . Data do not exist, -2 . Data exist but no retrieval, -1 . Clear sky, $i=0$. Valid cloud base height

7) CloudBaseMplCamp:

long_name: MPL Campbell et al. Algorithm Cloud Base Height

units: $\mathrm{m}$ AGL

comment: -3 . Data do not exist, -2 . Data exist but no retrieval, -1 . Clear sky, $i=0$. Valid cloud base height

8) CloudBaseMplCloth:

long_name: MPL Clothiaux et al. Algorithm Cloud Base Height

units: $\mathrm{m}$ AGL

comment: -3 . Data do not exist, -2 . Data exist but no retrieval, -1 . Clear sky, $i=0$. Valid cloud base height

\section{COMMENTS:}




\section{DATASTREAM: mmcrmode__v}

\section{CONTACT:}

\section{DIMENSIONS:}

1) time $=$ UNLIMITED

2) nheights $=256$

\section{VARIABLES:}

1) base_time:

long_name: Beginning Time of File units: seconds since 1970-01-01 00:00:00 00:00

calendar_date: Year 0000 Month 00 Day 00 00:00:00

2) time_offset:

long_name: Time Offset from base_time

units: seconds

comment: none

3) Heights:

long_name: Height of Measured Value units: $\mathrm{m}$ AGL

comment: none

\section{4) Reflectivity:}

long_name: MMCR Reflectivity

units: $\mathrm{dBZ}$

comment: none

5) MeanDopplerVelocity:

long_name: MMCR Mean Doppler Velocity

units: $\mathrm{m} / \mathrm{s}$

comment: none

6) SpectralWidth:

long_name: MMCR Spectral Width

units: $\mathrm{m} / \mathrm{s}$

comment: none

7) SignaltoNoiseRatio:

long_name: MMCR Signal-to-Noise Ratio

units: $\mathrm{dB}$

comment: none

8) CloudBasePrecipitation:

long_name: Microwave Radiometer Wet Window/Optical Rain Gauge Cloud Base Height units: $\mathrm{m}$ AGL

comment: -3 . Data do not exist, -2 . Data exist but no retrieval, -1 . Clear sky, $\dot{b}=0$. Valid cloud base height

9) CloudBaseCeilometerStd:

long_name: BLC/VCEIL Standard Algorithm Cloud Base Height units: $\mathrm{m}$ AGL

comment: -3 . Data do not exist, -2 . Data exist but no retrieval, -1 . Clear sky, $i=0$. Valid cloud base height

10) CloudBaseCeilometerCloth: 